\title{
MECHANISM DESIGN IN QUEUEING PROBLEMS *
}

\author{
Manipushpak Mitra \\ Revised on September 1999 \\ Indian Statistical Institute \\ 7, S. J. S. Sansanwal Marg \\ New Delhi-110016
}

*I wish to thank Debasish Goswami, Suresh Mutuswami, Sukanta Pati, Prof. Bhaskar Dutta, Prof. Arijit Sen, Prof. Jean Tirole and my advisor Prof. Arunava Sen for their invaluable advice. 


\begin{abstract}
A well-known result in incentive theory is that for a very broad class of decision problems, there is no mechanism which achieves truthtelling in dominant strategies, efficiency and budget balancedness (or first best implementability). On the contrary, Mitra and Sen (1998), prove that linear cost queueing models are first best implementable. This paper is an attempt at identification of cost structures for which queueing models are first best implementable. The broad conclusion is that, this is a fairly large class. Some of these first best implementable models can be implemented by mechanisms that satisfy individually rationality.
\end{abstract}




\section{Introductions}

There is a vast literature on incentive theory under incomplete information suggesting that under quasi-linear preferences the achievement of truthtelling and efficiency is possible. The pioneering work of Groves (1973) and Clarke (1971) has established that there exists a class of mechanisms, the so called Groves mechanisms where all individuals have a dominant strategy to reveal their information. Moreover, the truth-telling outcome leads to efficiency $^{1}$. However, Groves mechanisms are in general not balanced i.e there are preference realizations where aggregate transfers are non-zero. The budget imbalance of Groves mechanisms' in the context of public goods problem is shown in Green and Laffont (1977) ${ }^{2}$.

This paper develops and refines a line of research initiated by Mitra and Sen (1998). They show that there exists a class of "public decision problems" which are dominant strategy incentive compatible, efficient and balanced. One of the best examples of such a public decision problem is the queueing model which is the model we are concerned with. In this queueing model there is a server who has to serve a finite set of individuals. The server can serve one individual at a time. Thus, individuals have to wait in a queue. Waiting in a queue is costly for each individual. The server's objective is to order the individuals in a queue efficiently so as to minimise the aggregate waiting cost. If the cost of waiting in the queue is private information then an individual, if asked, will announce his costs strategically so as to get his job done as early as possible. Therefore, the principal in the queueing model has an incentive problem under incomplete information. A model similar to the queueing model is the sequencing model in Suijs (1996).

Mitra and Sen (1998) demonstrate that if waiting costs are linear, it is possible to devise a scheme of balanced transfers that induce individuals to

\footnotetext{
${ }^{1}$ Efficiency means that the outcome achieved in each state is the one that maximises the social surplus.

${ }^{2}$ See Green and Laffont (1979) for a comprehensive account of these mechanisms and their properties.
} 
reveal their private information and attain efficiency. Suijs (1996) proves a similar result in the context of his model. It is important to identify the reason why a possibility result holds in this model in contrast to the wellknown impossibility result in the case of the public good model. In the latter model, an individual who, by changing his announcement, affects the payoff all individuals. It is this severe nature of this externality that leads to budget imbalance (see Green and Laffont (1979)). In the queueing model with linear cost the externality that can be imposed by an individual is less severe and more subtle. An individual with $k$ th queue position by changing his announcement can affect the decision of either individuals who precede him in the queue or those who succeed him. He cannot simultaneously affect the decisions of both the predecessor and the successor sets. Thus only the individuals getting the first position and last position in the queue can affect all other individuals by changing their announcements. This sort of externality is necessary for finding a Groves transfer where the individuals served earlier compensates for those served later in such a way that aggregate transfer is zero in all states.

Another important feature of any queueing model is that the incentive problem is "spread over" the queue positions and this helps in finding a balanced Groves transfer where the individuals being served "earlier" pay money to the individuals receiving "late" service. For example, with three individuals, a balanced Groves transfer in the queueing model is of the following type. The individual receiving the service first pays the waiting cost of the individual who is served second in the queue and this money goes to the individual who is served last. The first part of the transfer resembles that of the transfer in second price auction where the highest bidder pays the second highest bid (see Vickrey (1961)). However, in the second price auction this money goes to an outsider like the principal. In the queueing model there is an incentive problem for all queue positions and so the payment of the second highest cost to the individual in the third queue position more then compensates him for the loss of getting the third queue position instead of 
the second queue position (see Mitra and Sen (1998)).

We attempt to answer the following question: are there cost structures more general than the linear case where the "first best" can be attained? We prove that for first best implementability it is both necessary and sufficient that preferences satisfy a certain combinatorial property and an independence property. The first property is a restriction on individual preferences while the second property is a restriction on the externality that an individual can impose on the remaining set of individuals. We need a combinatorial structure over the cost vector of each individual which is captured by the combinatorial property. Preferences satisfy the independence property if an individual, by changing his announcement, cannot change the relative queue positions of the remaining set of individuals. For instance, if there are $n$ individuals then the relative queue positions of any set of $(n-1)$ individuals are independent of the queue position of the individual who is left out. In spite of these requirements, apparently quite strong, there exists a fairly large class of queueing problems that are first best implementable. Given a broad class of first best implementable queueing models one can then explore the possibility of individual rationality i.e. whether individuals would be willing to participate in the mechanism offered by the server. It can be shown that if the gross benefit from the service for all individuals is sufficiently high, then first best implementable queueing models satisfy individual rationality.

This paper is organised as follows. In section two, the general queueing model is formalised and results on its first best implementability are derived. Section three deals with separable cost queueing models and its applications. Section four formalises a general class of first best implementable queueing problem. Section five is a discussion of discounted cost queueing model. The concluding section seven is preceded by an exploration of the possibility of individual rationality of first best queueing models in section six. 


\section{The General Model}

Let $\mathbf{N}=\{1,2, \ldots, n\}$ be the set of individuals and $\theta_{j}(k)$ measure the cost of waiting $k$ periods in the queue for individual $j$ where $k \in\{1, \ldots, n\}$. The type of individual $j \in \mathbf{N}$ is the vector $\theta_{j}=\left(\theta_{j}(1), \ldots, \theta_{j}(n)\right)$. Clearly, $\theta_{j}(k) \in \mathbf{R}_{+}$for all $j \in \mathbf{N}$ and for all $k \in\{1, \ldots, n\}^{3}$. It is assumed that all individuals dislike waiting i.e. $0 \leq \theta_{j}(1) \leq \theta_{j}(2) \leq \ldots \leq \theta_{j}(n)$. Let $\overline{\boldsymbol{\Theta}}$ be the largest domain satisfying this condition. For all $j \in \mathbf{N}, \theta_{j} \in \overline{\boldsymbol{\Theta}}$, the utility of each individual $j$ is assumed to be quasi-linear and is of the form:

$$
U_{j}\left(k, t_{j} ; \theta_{j}\right)=v_{j}-\theta_{j}(k)+t_{j}
$$

where $v_{j}(>0)$ is the gross benefit derived by individual $j$ from the service and $t_{j}$ is the transfer that individual $j$ receives.

The server's aim is to achieve efficiency or minimise the aggregate cost. A permutation $\sigma=\left(\sigma_{1}, \ldots, \sigma_{n}\right)$ of the set $\mathbf{N}$ represents a particular queue. Thus, $\sigma_{j}=k$ indicates that individual $j$ has the $k$ th position in the queue. Let $\Sigma$ be the set of all possible permutations of $\mathbf{N}$. Given a permutation or a queue $\sigma=\left(\sigma_{1}, \ldots, \sigma_{n}\right)(\in \Sigma)$, the cost of any individual $j \in \mathbf{N}$ is $\theta_{j}\left(\sigma_{j}\right)$. A state of the world is $\theta=\left(\theta_{1}, \ldots, \theta_{n}\right) \in \overline{\boldsymbol{\Theta}}^{n}$ where $\theta_{j}$ is a $1 \times n$ vector.

DEFINITION 2.1 Given a state $\theta$, a queue $\sigma^{*}=\left(\sigma_{1}^{*}, \ldots, \sigma_{n}^{*}\right)$ is efficient if $\sigma^{*} \in \operatorname{argmin}_{\sigma \in \Sigma} \sum_{j \in \mathbf{N}} \theta_{j}\left(\sigma_{j}\right)^{4}$.

Efficiency in this context is an assignment problem that gives each individual exactly one queue position and each queue position to exactly one individual in such a way that the aggregate cost is minimised ${ }^{5}$.

If the server knows $\theta=\left(\theta_{1}, \ldots, \theta_{n}\right)$ then he can calculate the efficient queue. However, if $\theta_{j}$ is private information for individual $j$, the server's

\footnotetext{
${ }^{3} \mathbf{R}_{+}$represents the non-negative orthant of $\mathbf{R}$.

${ }^{4}$ Observe that there can be states with more than one efficient queue. So we have an efficiency correspondence.

${ }^{5}$ This is a subtle optimization problem. An algorithm which computes efficiency is the Hungarian method which can be found in Bapat and Raghavan (1997).
} 
problem then is to design a mechanism that will elicit this information truthfully. Formally, a mechanism $\mathbf{M}$ is a pair $\langle\sigma, \mathbf{t}\rangle$ where $\sigma: \overline{\boldsymbol{\Theta}}^{n} \rightarrow \Sigma$ and $\mathbf{t} \equiv\left(t_{1}, \ldots, t_{n}\right): \overline{\boldsymbol{\Theta}}^{n} \rightarrow \mathbf{R}^{n}$. This problem is called a general queueing problem under incomplete information and is written as $\Omega=\langle\mathbf{N}, \overline{\boldsymbol{\Theta}}\rangle$. Under $\mathbf{M}=\langle\sigma, \mathbf{t}\rangle$, given all others' announcement $\theta_{-j}$, the utility of individual $j$ of type $\theta_{j}$ when his announcement is $\theta_{j}{ }^{\prime}$ is given by $U_{j}\left(\sigma_{j}\left(\theta_{j}{ }^{\prime}, \theta_{-j}\right), t_{j}\left(\theta_{j}{ }^{\prime}, \theta_{-j}\right), \theta_{j}\right)=v_{j}-\theta_{j}\left(\sigma_{j}\left(\theta_{j}{ }^{\prime}, \theta_{-j}\right)\right)+t_{j}\left(\theta_{j}{ }^{\prime}, \theta_{-j}\right)$.

DEFINITION 2.2 $\Omega=\langle\mathbf{N}, \overline{\boldsymbol{\Theta}}\rangle$ is implementable if there exists an efficient rule $\sigma^{6} \sigma^{*}: \overline{\boldsymbol{\Theta}}^{n} \rightarrow \boldsymbol{\Sigma}$ and a mechanism $\mathbf{M}=\left\langle\sigma^{*}, \mathbf{t}\right\rangle$ such that for all $j \in \mathbf{N}$, for all $\left(\theta_{j}, \theta_{j}{ }^{\prime}\right) \in \overline{\mathbf{\Theta}}^{2}$, and for all $\theta_{-j} \in \overline{\mathbf{\Theta}}^{n-1}$,

$$
U_{j}\left(\sigma_{j}^{*}(\theta), t_{j}(\theta) ; \theta_{j}\right) \geq U_{j}\left(\sigma_{j}^{*}\left(\theta_{j}{ }^{\prime}, \theta_{-j}\right), t_{j}\left(\theta_{j}{ }^{\prime}, \theta_{-j}\right) ; \theta_{j}\right)
$$

This definition says that for any given $\theta_{-j}$, individual $j$ cannot benefit by reporting anything other than his true type. In other words, truth-telling is a dominant strategy for all individuals. Moreover, this truth-telling leads to efficient queue.

DEFINITION 2.3 $\Omega=\langle\mathrm{N}, \overline{\mathrm{\Theta}}\rangle$ is first best implementable or FB implementable, if there exists a mechanism $\mathbf{M}=\left\langle\sigma^{*}, \mathbf{t}\right\rangle$ which implements it and such that, for all $\theta \in \overline{\mathbf{\Theta}}^{n}, \sum_{j \in \mathbf{N}} t_{j}(\theta)=0$.

Thus, a queueing problem is first-best implementable if, it can be implemented in a manner such that aggregate transfers are zero in every state of the world. In such problems, incomplete information does not impose any welfare cost. In the next section the question of FB implementability of the general queueing model is analysed.

\subsection{Characterization Results}

In this sub-section the necessary and sufficient conditions relating to the FB implementability of the general queueing model are derived. As a preliminary

\footnotetext{
${ }^{6} \mathrm{An}$ efficient rule is a single valued selection from the efficiency correspondence.
} 
step to the main result, some more definitions and notations are introduced that will be extensively used in this section.

DEFINITION 2.4 A mechanism $\mathbf{M}=\langle\sigma, \mathbf{t}\rangle$ is a Groves mechanism if for all $j \in \mathbf{N}$, the transfer is of the form

$$
t_{j}(\theta)=-\sum_{l \neq j} \theta_{l}\left(\sigma_{l}^{*}(\theta)\right)+\gamma_{j}\left(\theta_{-j}\right)
$$

In a Groves mechanism, the transfer of any individual $j \in \mathbf{N}$ in any state $\theta$ is the negative of minimum cost i.e. $-\sum_{l \in \mathbf{N}} \theta_{l}\left(\sigma_{l}^{*}(\theta)\right)$ plus the cost of individual $j$ and a constant $\gamma_{j}\left(\theta_{-j}\right)$. The utility of individual $j$ with a Groves transfer is his gross benefit $v_{j}$ less the minimum cost in state $\theta$ plus the constant. It is well known that such a transfer results in dominant strategy incentive compatibility because the servers' objective of minimising the aggregate cost is now an objective of individual $j$ as well and this is true for all $j \in \mathbf{N}$.

According to a well known result of Holmström (see Holmström (1979)), decision problems with "smoothly connected" domains are implementable if and only if the mechanism is a Groves mechanism. In more precise terms, convex domains are "smoothly connected" (see Theorem (2) in Holmström (1979)). It can be easily checked that the domain under consideration in the general queueing model satisfy Holmström's definition of "convex" domains. Hence it is implementable if and only if the mechanism is a Groves mechanism.

Let $C\left(\sigma^{*}\left(\theta^{\prime}\right) ; \theta\right)=\sum_{j \in \mathbf{N}} \theta_{j}\left(\sigma_{j}^{*}\left(\theta^{\prime}\right)\right)$ where, as stated earlier, $\sigma^{*}\left(\theta^{\prime}\right)$ is an efficient queue for the announced state $\theta^{\prime}$. Thus, $C\left(\sigma^{*}\left(\theta^{\prime}\right) ; \theta\right)$ is the minimum aggregate cost with respect to the announced state $\theta^{\prime}$ when the actual state is $\theta$. For notational simplicity define $C(\theta) \equiv C\left(\sigma^{*}(\theta) ; \theta\right)$ to be the minimum aggregate cost with respect to the actual state $\theta$ when the announced state is also $\theta$.

REMARK 2.1 From the definition of efficiency it follows that for all $\theta$ and $\theta^{\prime}, C(\theta) \leq C\left(\sigma^{*}\left(\theta^{\prime}\right) ; \theta\right)$. 
DEFINITION 2.5 $\Omega=\langle\mathbf{N}, \bar{\Theta}\rangle$ satisfies the Combinatorial Property (or CP) if for all $j \in \mathbf{N}$, for all $\theta_{j} \in \overline{\mathbf{\Theta}}$,

$$
\sum_{k=1}^{n}(-1)^{k-1}\left(\begin{array}{l}
n-1 \\
k-1
\end{array}\right) \theta_{j}(k)=0
$$

This property is a combinatorial condition on the domain of preferences. The meaning of this property will become explicit from the following discussion. For individual $j$ with type $\theta_{j}=\left(\theta_{j}(1), \ldots, \theta_{j}(n)\right)$ define the first order difference at queue position $k \in\{1, \ldots, n-1\}$ as $\Delta(1) \theta_{j}(k)=\theta_{j}(k+1)-\theta_{j}(k)$. Thus, the first order difference at $k$ represents the increase in queueing cost if individual $j$ is moved from $k$ th position to $(k+1)$ th position. In particular, the first order difference at queue position 1 is $\Delta(1) \theta_{j}(1)=\theta_{j}(2)-\theta_{j}(1)$. Similarly, the second order difference at queue position 1 is $\Delta^{2}(1) \theta_{j}(1)=$ $\Delta(1)\left[\Delta(1) \theta_{j}(1)\right]=\Delta(1)\left[\theta_{j}(2)-\theta_{j}(1)\right]=\theta_{j}(3)-2 \theta_{j}(2)+\theta_{j}(1)$. One can similarly derive $\Delta^{3}(1) \theta_{j}(1), \Delta^{4}(1) \theta_{j}(1)$ and so on. It can be quite easily checked from (2.2) that

$$
\sum_{k=1}^{n}(-1)^{k-1}\left(\begin{array}{l}
n-1 \\
k-1
\end{array}\right) \theta_{j}(k)=\Delta^{n-1}(1) \theta_{j}(1)=0 .
$$

Thus a type $\theta_{j}$ of individual $j$ satisfies CP if the $(n-1)$ th order difference at queue position 1 is zero. CP is analogous to $(n-1)$ th order derivative at queue position 1. CP implies and is implied by some kind of separability to be discussed later in Proposition 2.1.

To define the next property one needs to introduce some more notations and definitions. Consider a queueing model $\Omega$. Define, by eliminating $l \in \mathbf{N}$, the $l$-reduced queueing model of $\Omega$ to be $\Omega_{N-l}=\langle\mathbf{N}-l, \overline{\mathbf{\Theta}}\rangle$. In any state $\theta_{-l} \in \Theta^{n-1}$, let $\sigma^{*}\left(\theta_{-l}\right)$ be the efficient queue in $\Omega_{N-l}$. In other words,

$$
\sigma^{*}\left(\theta_{-l}\right) \in \operatorname{argmin}_{\tilde{\sigma} \in \Sigma(N-l)} \sum_{j \neq l} \theta_{j}\left(\tilde{\sigma}_{j}\right)
$$

where $\Sigma(N-l)$ is the set of all possible permutations of $\{1, \ldots, n-1\}$ and $\tilde{\sigma}_{j}$ is the position of individual $j(\neq l)$ in the particular queue $\tilde{\sigma} \in \Sigma(N-l)$. 
In short, $\Omega_{N-l}$ is a l-reduced queueing model of $(n-1)$ individuals obtained from $\Omega$ by excluding $l \in \mathbf{N}$.

For $\Omega$, define $P\left(\sigma^{*}(\theta), j\right)=\left\{p \in \mathbf{N} /\{j\} \mid \sigma_{j}^{*}(\theta)>\sigma_{p}^{*}(\theta)\right\}$ to be the predecessor set of individual $j$ in state $\theta$. In other words, under the efficient queue $\sigma^{*}(\theta)$ in state $\theta, P\left(\sigma^{*}(\theta), j\right)$ is the set of individuals receiving the service before individual $j$. Similarly, for $\Omega_{N-l}$, define $P\left(\sigma^{*}\left(\theta_{-l}\right), j\right)=\{p \in$ $\left.\mathbf{N} /\{j, l\} \mid \sigma_{j}^{*}\left(\theta_{-l}\right)>\sigma_{p}^{*}\left(\theta_{-l}\right)\right\}$ to be the predecessor set of individual $j$ in state $\theta_{-l}$. Under the efficient queue $\sigma^{*}\left(\theta_{-l}\right)$ in state $\theta_{-l}, P\left(\sigma^{*}\left(\theta_{-l}\right), j\right)$ is the set of individuals receiving the service before individual $j$.

DEFINITION 2.6 $\Omega$ satisfies Independence Property (or IP) if for all states $\theta \in \overline{\mathbf{\Theta}}^{n}$, for all $j \in \mathbf{N}$, for all $l \in \mathbf{N} /\{j\}$ and for all $\theta_{-l} \in \overline{\mathbf{\Theta}}^{n-1}$,

$$
P\left(\sigma^{*}\left(\theta_{-l}\right), j\right)= \begin{cases}P\left(\sigma^{*}(\theta), j\right) & \text { if } l \notin P\left(\sigma^{*}(\theta), j\right) \\ P\left(\sigma^{*}(\theta), j\right) /\{l\} & \text { if } l \in P\left(\sigma^{*}(\theta), j\right)\end{cases}
$$

This property means that if in state $\theta, \sigma_{j}^{*}(\theta)=k$, then $\sigma_{j}^{*}\left(\theta_{-l}\right)=k$ for all $l \notin P\left(\sigma^{*}(\theta), j\right)$ and $\sigma_{j}^{*}\left(\theta_{-l}\right)=k-1$ for all $l \in P\left(\sigma^{*}(\theta), j\right)$. Another way of stating IP is the following: Consider $\Omega$ and a pair $\{j, l\} \in \mathbf{N}$. If $\sigma_{j}^{*}(\theta)<\sigma_{l}^{*}(\theta)$ in state $\theta$, then $\sigma_{j}^{*}\left(\theta_{-i}\right)<\sigma_{l}^{*}\left(\theta_{-i}\right)$ for all $i \in \mathbf{N} /\{j, l\}$. If this condition holds for all pair of individuals and for all states in $\Omega$ then it is easy to check that $\Omega$ satisfies IP. This condition says that if individual $j$ 's position in the queue is less than that of individual $l$ in some state $\theta$, then $j$ 's queue position must continue to remain less than that of $l$ 's position in all $\Omega_{N-i}$ that includes both $j$ and $l$. This condition must hold for all pair $\{j, l\} \in \mathbf{N}$ and for all states $\theta \in \overline{\mathbf{\Theta}}^{n}$. IP eliminates the possibility that an individual $l \in \mathbf{N} /\{j\}$, who is a predecessor (successor) of individual $j$ in state $\theta$ is a successor (predecessor) of individual $j$ in state $\theta_{-i}$ for some $i$ reduced queueing model $\Omega_{N-i}$ where $i \in \mathbf{N} /\{j, l\}$. Thus, IP guarantees that the externality imposed by an individual ( $i$ in the above argument) is not severe enough to change the relative queue position of the remaining set of individuals. 
The separability implied by the combinatorial property and the link between the combinatorial property (or CP) and the independence property (or IP) is captured by the following Proposition and the explanation following it.

PROPOSITION 2.1 $\Omega=\langle\mathbf{N}, \overline{\boldsymbol{\Theta}}\rangle$ satisfies CP, if and only if for each $\theta_{j} \in$ $\bar{\Theta}$ there exists a unique vector $H_{j}=\left\{h_{j}(1), \ldots, h_{j}(n-1)\right\}$ such that for all $k \in\{1, \ldots, n\}$,

$$
\theta_{j}(k)=(n-k) h_{j}(k)+(k-1) h_{j}(k-1) .
$$

PROOF: Given a $n \times 1$ vector of type for individual $j \in \mathbf{N}, \theta_{j} \in \overline{\boldsymbol{\Theta}}$ in $\Omega$ satisfying $\mathrm{CP}$, we define a $(n-1) \times 1$ vector $H_{j}=\left\{h_{j}(1), \ldots, h_{j}(n-1)\right\}$ such that for all $k \in\{1, \ldots, n-1\}$,

$$
h_{j}(k)=\sum_{r=1}^{k}(-1)^{k-r} \frac{(k-1) !(n-k-1) !}{(r-1) !(n-r) !} \theta_{j}(r)
$$

First we check using (2.4) that for all $k \in\{1, \ldots, n-1\},(2.1)$ holds. Then we check for $k=n$ this condition holds only if $\Omega$ satisfies CP.

$(n-k) h_{j}(k)+(k-1) h_{j}(k-1)$

$=(n-k) \sum_{r=1}^{k}(-1)^{k-r} \frac{(k-1) !(n-k-1) !}{(r-1) !(n-r) !} \theta_{j}(r)+(k-1) \sum_{r=1}^{k-1}(-1)^{k-r-1} \frac{(k-2) !(n-k) !}{(r-1) !(n-r) !} \theta_{j}(r)$

$=\sum_{r=1}^{k-1}\left\{(-1)^{k-r}+(-1)^{k-r-1}\right\} \frac{(k-1) !(n-k) !}{(r-1) !(n-r) !} \theta_{j}(r)+\theta_{j}(k)$

$=\theta_{j}(k)$ (because $\left.(-1)^{k-r}+(-1)^{k-r-1}=0\right)$.

For $k=n$,

$(n-k) h_{j}(k)+(k-1) h_{j}(k-1)$

$=(n-1) h_{j}(n-1)$

$=(n-1) \sum_{r=1}^{n-1}(-1)^{n-1-r} \frac{(n-2) !}{(r-1) !(n-r) !} \theta_{j}(r)$

$=\sum_{r=1}^{n-1}(-1)^{n-1-r} \frac{(n-1) !}{(r-1) !(n-r) !} \theta_{j}(r)$ 
$=\sum_{r=1}^{n-1}(-1)^{n-1-r}\left(\begin{array}{c}n-1 \\ r-1\end{array}\right) \theta_{j}(r)$

$=\theta_{j}(n)($ from $\mathrm{CP})$.

The last step not only proves the necessity of CP but also guarantees that for $\theta_{j}, H_{j}$ is unique.

To prove the other part of the Lemma it is easy to see that if $\theta_{j}(k)=$ $(n-k) h_{j}(k)+(k-1) h_{j}(k-1)$ for all $k=1, \ldots, n$ then

$\sum_{k=1}^{n}(-1)^{k-1}\left(\begin{array}{l}n-1 \\ k-1\end{array}\right) \theta_{j}(k)$

$=\sum_{k=1}^{n}(-1)^{k-1}\left(\begin{array}{l}n-1 \\ k-1\end{array}\right)\left\{(n-k) h_{j}(k)+(k-1) h_{j}(k-1)\right\}$

$=(n-1)\left\{\sum_{k=1}^{n-1}(-1)^{k-1}\left(\begin{array}{l}n-2 \\ k-1\end{array}\right) h_{j}(k)+\sum_{k=2}^{n}(-1)^{k-1}\left(\begin{array}{l}n-2 \\ k-2\end{array}\right) h_{j}(k-1)\right\}$

$=0$.

Consider $\Omega$ and some state $\theta$ where individual $j \in \mathbf{N}$ gets the $k$ th queue position. Note that in state $\theta$, out of the remaining $(n-1)$ individuals in $\mathbf{N} /\{j\}$, there are $(n-k)$ individuals receiving the service after individual $j$ and there are $(k-1)$ individuals receiving the service before individual $j$. Consider $\Omega_{N-l}$ for all $l \in \mathbf{N} /\{j\}$. Now if an individual $l$ receiving the service after individual $j$, i.e. $l \notin P\left(\sigma^{*}(\theta), j\right)$, were to be eliminated, then from IP it follows that $j$ retains the $k$ th queue position in $\Omega_{N-l}$. If, on the other hand, an individual $l$ who was receiving the service before individual $j$, i.e. $l \in P\left(\sigma^{*}(\theta), j\right)$, is eliminated from the queue then, from IP it follows that the queue position of individual $j$ changes from $k$ in $\Omega$ to $(k-1)$ in $\Omega_{N-l}$. If the vector $H_{j}$ in Proposition 2.1 replaces $\theta_{j}$ for reduced queueing models $\left\{\Omega_{N-l}\right\}_{l \neq j}$, the cost of $k$ th queue position for individual $j$ i.e. $\theta_{j}(k)$, in $\Omega$ for state $\theta$, can now be represented as the sum of costs in $(n-1)$ reduced queueing models. Here individual $j$ has cost $h_{j}(k)$ in $(n-k)$ of the reduced queueing models. These are reduced models $\Omega_{N-l}$ such that $l \notin P\left(\sigma^{*}(\theta), j\right)$ and total number of such reduced models is $\left|\mathbf{N}-\left[P\left(\sigma^{*}(\theta), j\right) \cup\{j\}\right]\right|=n-k$. Similarly, individual $j$ has a cost of $h_{j}(k-1)$ in $(k-1)$ of these models. These are reduced models $\Omega_{N-l}$ such that $l \in P\left(\sigma^{*}(\theta), j\right)$ and total number 
of such reduced models is $\left|P\left(\sigma^{*}(\theta), j\right)\right|=k-1$. Observe that this will give $\theta_{j}(k)=(n-k) h_{j}(k)+(k-1) h_{j}(k-1)$ which follows from CP as established in Proposition 2.1.

REMARK 2.2 Consider an individual $j$ and a profile $\theta_{j} \in \overline{\boldsymbol{\Theta}}$ satisfying CP. From Proposition 2.1 it follows that there exists a unique vector $H_{j}$ such that for all $k \in\{1, \ldots, n\}, \theta_{j}(k)=(n-k) h_{j}(k)+(k-1) h_{j}(k-1)$. Using $\theta_{j}(1) \leq \theta_{j}(2) \leq \ldots \leq \theta_{j}(n)$ one obtains the following restriction on the elements of $H_{j} . h_{j}(1) \leq h_{j}(r) \leq h_{j}(n-1)$ for all $r \in\{2, \ldots, n-2\}$. One cannot comment on the ordering of the set of elements belonging to the set $\left\{h_{j}(2), \ldots, h_{j}(n-2)\right\}$.

REMARK 2.3 Consider $\Omega$ and $\left\{\theta_{j}, \theta_{l}\right\} \in \overline{\boldsymbol{\Theta}}^{2}$ such that $\theta_{j}(k+1)-\theta_{j}(k) \geq$ $\theta_{l}(k+1)-\theta_{l}(k)$ for all $k \in\{1, \ldots, n\}$. Note that $\theta_{j}(k+1)-\theta_{j}(k) \geq$ $\theta_{l}(k+1)-\theta_{l}(k)$ implies that if in some state individuals $j$ and $l$ are assigned queue positions $k$ and $k+1$, then it is more efficient to serve individual $j$ ahead of individual $l$ because the marginal cost of shifting individual $j$ from queue position $k$ to queue position $k+1$ is no less than the same marginal cost for individual $l$. If this condition is true for all $k$ then $\sigma_{j}^{*}\left(\theta_{j}, \theta_{l}, \theta_{-j-l}\right)<\sigma_{l}^{*}\left(\theta_{j}, \theta_{l}, \theta_{-j-l}\right)$ for all $\theta_{-j-l} \in \overline{\mathbf{\Theta}}^{n-2}$. One obvious implication of this observation is that $\sigma_{j}^{*}\left(\theta_{j}, \theta_{l}, \theta_{-j-l-i}\right)<\sigma_{l}^{*}\left(\theta_{j}, \theta_{l}, \theta_{-j-l-i}\right)$ for all $i \in \mathbf{N} /\{j, l\}$ and for all $\theta_{-j-l-i} \in \overline{\boldsymbol{\Theta}}^{n-3}$. Another useful implication is the following. Consider a state $\theta$ where for all pairs $\{j, l\}$, if $\theta_{j}(2)-\theta_{j}(1) \geq \theta_{l}(2)-\theta_{l}(1)$, then $\theta_{j}(k+1)-\theta_{j}(k) \geq \theta_{l}(k+1)-\theta_{l}(k)$ for all $k \in\{2, \ldots, n-1\}$. In state $\theta$, if for some pair $\{j, l\}, \sigma_{j}^{*}(\theta)<\sigma_{l}^{*}(\theta)$ then from the construction of state $\theta$ it follows that $\sigma_{j}^{*}\left(\theta_{-i}\right)<\sigma_{l}^{*}\left(\theta_{-i}\right)$ for all $i \in \mathbf{N} /\{j, l\}$. Therefore, in state $\theta, \Omega$ satisfies IP. This remark will be used in some of the results to follow.

In the case of $|\mathbf{N}|=3$, it is possible to show that CP implies IP. Unfortunately, for $|\mathbf{N}|>3$ this is no longer true.

PROPOSITION 2.2 $\Omega=\langle\mathbf{N}=\{1,2,3\}, \overline{\mathbf{\Theta}}\rangle$ satisfies CP $\Rightarrow \Omega$ satisfies IP. 
PROOF: $\Omega=\langle\mathbf{N}=\{1,2,3\}, \overline{\boldsymbol{\Theta}}\rangle$ satisfies CP implies that the second order difference is zero. Thus $\Delta(1) \theta_{j}(1) \equiv \theta_{j}(2)-\theta_{j}(1)=\theta_{j}(3)-\theta_{j}(2) \equiv \Delta(1) \theta_{j}(2)$ for all $j=1,2,3$. Therefore, for a pair of preferences $\left\{\theta_{j}, \theta_{l}\right\} \in \overline{\boldsymbol{\Theta}}^{2}$, if $\theta_{j}(2)-\theta_{j}(1) \geq \theta_{l}(2)-\theta_{l}(1)$, then $\theta_{j}(3)-\theta_{j}(2) \geq \theta_{l}(3)-\theta_{l}(2)$. Using Remark 2.3 it immediately follows that $\Omega$ satisfies IP.

The next example is to show that for $|\mathbf{N}|>3$, there is no relationship between CP and IP. Specifically, it shows that if $\Omega$ satisfies CP, it may not satisfy IP.

EXAMPLE 2.1 Consider the general queueing model for four individuals, i.e. $\Omega=\langle\mathbf{N}=\{1,2,3,4\}, \overline{\mathbf{\Theta}}\rangle$. Let the state $\theta=\left(\theta_{1}, \theta_{2}, \theta_{3}, \theta_{4}\right)$ be of the following form: $\theta_{1}=(1,23,45,67), \theta_{2}=(3,12,27,48), \theta_{3}=(1,4,9,16)$ and $\theta_{4}=(1,7,13,19)$. Here, $\sigma_{j}^{*}(\theta)=j$ for all $j=1,2,3,4$. We find that state $\theta$ satisfies CP i.e. for all $j=1,2,3,4$,

$\sum_{k=1}^{4}(-1)^{k-1}\left(\begin{array}{c}3 \\ k-1\end{array}\right) \theta_{j}(k)=\left(\begin{array}{l}3 \\ 0\end{array}\right) \theta_{j}(1)-\left(\begin{array}{l}3 \\ 1\end{array}\right) \theta_{j}(2)+\left(\begin{array}{l}3 \\ 2\end{array}\right) \theta_{j}(3)-\left(\begin{array}{l}3 \\ 3\end{array}\right) \theta_{j}(4)=0$.

Now consider the $\{1\}$-reduced queueing model $\Omega_{N-1}=\langle\{2,3,4\}, \overline{\boldsymbol{\Theta}}\rangle$. In this reduced model we consider the first three elements of the vectors $\theta_{2}, \theta_{3}$ and $\theta_{4}$. Here $\sigma_{2}^{*}\left(\theta_{-1}\right)=1, \sigma_{3}^{*}\left(\theta_{-1}\right)=3$ and $\sigma_{4}^{*}\left(\theta_{-1}\right)=2$. Therefore, $P\left(\sigma^{*}\left(\theta_{-1}\right), 3\right)=\{2,4\} \neq P\left(\sigma^{*}(\theta), 3\right) /\{1\}=\{2\}$ and $P\left(\sigma^{*}\left(\theta_{-1}\right), 4\right)=\{2\} \neq$ $P\left(\sigma^{*}(\theta), 4\right) /\{1\}=\{2,3\}$. Thus for state $\theta$, IP is violated.

THEOREM 2.1 $\Omega=\langle\mathbf{N}, \overline{\mathbf{\Theta}}\rangle$ is FB implementable if only if it satisfies $\mathrm{CP}$ and IP.

Before proving the Theorem a Lemma due to Walker (1980) is stated below. Consider two profiles $\theta=\left(\theta_{1}, \ldots, \theta_{n}\right)$ and $\theta^{\prime}=\left(\theta_{1}^{\prime}, \ldots, \theta_{n}^{\prime}\right)$. Define for $S \subseteq \mathbf{N}$, a type $\theta_{j}(S)=\theta_{j}$ if $j \notin S$ and $\theta_{j}(S)=\theta_{j}^{\prime}$ if $j \in S$. Thus for each $S \subseteq \mathbf{N}$, we have a state $\theta(S)=\left(\theta_{1}(S), \ldots, \theta_{n}(S)\right)$.

LEMMA 2.1 $\Omega$ is FB implementable only if for all $\left(\theta, \theta^{\prime}\right) \in \overline{\boldsymbol{\Theta}}^{n} \times \overline{\mathbf{\Theta}}^{n}$, $\sum_{S \subseteq \mathbf{N}}(-1)^{|S|} C(\theta(S))=0$. 
It is quite easy to see why Lemma 2.1 is necessary for FB implementability. Given the Groves transfer, balancedness requires that $(n-1) C(\theta)=$ $\sum_{j \in \mathbf{N}} \gamma_{j}\left(\theta_{-j}\right)^{7}$. For any two profiles $\theta$ and $\theta^{\prime}$ one can now easily check that $\sum_{S \subseteq \mathbf{N}}(-1)^{|S|} C(\theta(S))=\frac{1}{(n-1)} \sum_{j \in \mathbf{N}} \sum_{S \subseteq \mathbf{N}}(-1)^{|S|} \gamma_{j}\left(\theta_{-j}(S)\right)=0$. It is quite clear that if the function $C$ has a separable form , then it must satisfy an appropriate restriction on the $n$th order cross partial derivative. The condition in the Lemma is analogous of this derivative for finite changes.

\section{PROOF OF THEOREM(2.1):}

Necessity: We prove the necessity part of the Theorem in two steps. In the first step we prove that $\Omega$ is FB implementable only if it satisfies CP. In the second step we prove that $\Omega$ satisfying $\mathrm{CP}$ is FB implementable only if it satisfies IP.

Step 1: We start with a given type for individual 1 (i.e. $\theta_{1}$ ) and construct $\theta_{-1}$ and $\theta^{\prime}$. Then we apply Lemma 2.1 to derive the result. Consider individual 1 and any announcement $\theta_{1}=\left(\theta_{1}(1), \ldots, \theta_{1}(k), \ldots, \theta_{n}(n)\right)$. Define real numbers $\left\{\epsilon_{1}, \epsilon_{2}, \ldots, \epsilon_{n}, \underline{\theta}\right\}$ such that $0=\epsilon_{1}<\epsilon_{2}<\ldots<\epsilon_{n}$ and $0 \leq \underline{\theta} \leq \theta_{1}(1)$. Consider two states $\theta=\left(\theta_{1}, \ldots, \theta_{n}\right)$ and $\theta^{\prime}=\left(\theta_{1}^{\prime}, \ldots, \theta_{n}^{\prime}\right)$ of the following type: $\theta_{j}(k)=\theta_{1}(k)+k \epsilon_{j}$ and $\theta_{j}^{\prime}(k)=\underline{\theta}$, for all $j \in \mathbf{N}$ and for all $k=1, \ldots, n$. Therefore $\theta_{j}=\left(\theta_{1}(1)+\epsilon_{j}, \theta_{1}(2)+\epsilon_{j}, \ldots, \theta_{1}(n)+n \epsilon_{j}\right)$ and $\theta_{j}^{\prime}=(\underline{\theta}, \underline{\theta}, \ldots, \underline{\theta})$ for all $j \in \mathbf{N}$. Consider any two queue positions $k$ and $k+1$ and any two individuals $j$ and $j+1$ with types $\theta_{j}$ and $\theta_{j+1}$, respectively. Note that from the construction of $\theta$, on the one hand, it follows that if individual $j$ gets the $k$ th position and $(j+1)$ th individual gets the $(k+1)$ th position, then the costs for these two positions add up to $\left\{2 \theta_{1}(k)+k \epsilon_{j}+(k+1) \epsilon_{j+1}\right\}$. If, on the other hand, the positions of $j$ and $(j+1)$ are interchanged then the costs add up to $\left\{2 \theta_{1}(k)+(k+1) \epsilon_{j}+k \epsilon_{j+1}\right\}$. Clearly the former cost exceeds the latter ${ }^{8}$ and holds for all $k=1, \ldots, n-1$. Thus the queue that minimises the aggregate cost requires that, $\sigma_{j}^{*}(\theta)>\sigma_{j+1}^{*}(\theta)$

\footnotetext{
${ }^{7}$ Adding the Groves transfer of all individuals and setting it to zero gives this condition. This condition in a more general framework was derived by Holmström (1977).

${ }^{8}$ This is because from the construction it follows that $\epsilon_{j+1}>\epsilon_{j}$ for all $j=1, \ldots n-1$.
} 
for all $j=1, \ldots, n-1$. This implies that the efficient queue in state $\theta$ is $\sigma^{*}(\theta)=\left(\sigma_{1}^{*}(\theta)=n, \ldots, \sigma_{j}^{*}(\theta)=n-j+1, \ldots, \sigma_{n}^{*}(\theta)=1\right)$. In state $\theta^{\prime}$ any queue is efficient because the costs of all individuals are identical.

Now consider profiles $\theta(S)=\left(\theta_{1}(S), \ldots, \theta_{n}(S)\right)$ where $\theta_{j}(S)=\theta_{j}$ if $j \notin S$ and $\theta_{j}(S)=\theta_{j}^{\prime}$ if $j \in S$. For all $s \in S$, the efficient queue position is behind all $j \notin S$, i.e. $\sigma_{s}^{*}(\theta(S)) \in\{n-|S|+1, \ldots, n\}$. This is because the queueing costs of all individuals $j \notin S$, in all queue positions strictly exceed the queueing costs of all individual $s \in S$. Moreover, given $\theta_{1}$, from the construction of $\theta_{-1}$ and from the argument given for the efficient queue in state $\theta$ it follows that if $\{j, l\} \notin S$ and $j<l$, then $\sigma_{j}^{*}(\theta(S))>\sigma_{l}^{*}(\theta(S))$.

Consider the sum $\sum_{S \subseteq \mathbf{N}}(-1)^{|S|} C(\theta(S))$. Observe first that, for all $l \in$ $\mathbf{N} /\{1\}$ with type $\theta_{l}$, if there exists $m$ sets $T^{1}, \ldots, T^{m}$ of size $\left|T^{1}\right|, \ldots,\left|T^{m}\right|$ respectively with $T^{\hat{m}} \subseteq \mathbf{N} /\{l\}$ for all $\hat{m}=1, \ldots, m$, for which individual l's position is $k(\in\{1,2, \ldots, n\})$, then $\sum_{\hat{m}=1}^{m}(-1)^{\left|T^{\hat{m}}\right|} \theta_{l}(k)=0$. Therefore, the sum $\sum_{S \subseteq \mathbf{N}}(-1)^{|S|} C(\theta(S))$ is independent of all elements in the set of vectors $\left\{\theta_{2}, \ldots, \theta_{n}\right\}$. Also observe that the terms containing $\underline{\theta}$ in $\sum_{S \subseteq \mathbf{N}}(-1)^{|S|} C(\theta(S))$ is given by $-n\left\{\sum_{k=1}^{n}(-1)^{k-1}\left(\begin{array}{l}n-1 \\ k-1\end{array}\right)\right\} \underline{\theta}=0$. Therefore, $\sum_{S \subseteq \mathbf{N}}(-1)^{|S|} C(\theta(S))$ is also independent of $\underline{\theta}$. All these observations imply that:

$\sum_{S \subseteq \mathbf{N}}(-1)^{|S|} C(\theta(S))=\sum_{S \subseteq \mathbf{N} /\{1\}}(-1)^{|S|} \theta_{1}\left(\sigma_{1}^{*}\left(\theta_{1}, \theta_{-1}(S)\right)\right)$.

For individual 1 with type $\theta_{1}, \sigma_{1}^{*}\left(\theta_{1}, \theta_{-1}(S)\right)=n-|S|$ for all $S \subseteq \mathbf{N} /\{1\}$. Thus

$$
\begin{aligned}
& \sum_{S \subseteq \mathbf{N} /\{1\}}(-1)^{|S|} \theta_{1}\left(\sigma_{1}^{*}\left(\theta_{1}, \theta_{-1}(S)\right)\right) \\
& =\sum_{|S|=0}^{n-1}(-1)^{|S|}\left(\begin{array}{c}
n-1 \\
|S|
\end{array}\right) \theta_{1}(n-|S|) \\
& =\sum_{k=1}^{n}(-1)^{k-1}\left(\begin{array}{c}
n-1 \\
k-1
\end{array}\right) \theta_{1}(n-k+1) \\
& =\sum_{k=1}^{n}(-1)^{n-k}\left(\begin{array}{c}
n-1 \\
n-k
\end{array}\right) \theta_{1}(k)
\end{aligned}
$$


$=(-1)^{n-2}\left\{\sum_{k=1}^{n}(-1)^{k-1}\left(\begin{array}{l}n-1 \\ k-1\end{array}\right) \theta_{1}(k)\right\}$.

An application of Lemma 2.1 yields the result for individual 1. We can obtain the same result for all $j \in \mathbf{N} /\{1\}$.

Step 2: For $|\mathbf{N}|=3, \Omega$ satisfying CP is FB implementable only if it satisfies IP follows from Proposition 2.2. For $\Omega$ with $|\mathbf{N}|>3$, consider any two cost vectors $\theta_{1}=\left(\theta_{1}(1), \ldots, \theta_{1}(n)\right)$ and $\theta_{2}=\left(\theta_{2}(1), \ldots, \theta_{2}(n)\right)$ for individuals 1 and 2 respectively. Let $z=\max \left[\max \left\{\Delta(1) \theta_{1}(k)\right\}_{k \neq n}, \max \left\{\Delta(1) \theta_{2}(k)\right\}_{k \neq n}\right]$. Consider the real numbers $\left\{\epsilon_{3}, \ldots, \epsilon_{n}, \underline{\theta}\right\}$ such that $z<\epsilon_{3}<\ldots<\epsilon_{n}$ and $\underline{\theta} \in\left[0, \min \left\{\theta_{1}(1), \theta_{2}(1)\right\}\right]$. Define $\theta_{j}(k)=k \epsilon_{j}$ for all $k \in\{1, \ldots, n\}$ and for all $j \in\{3, \ldots, n\}$. Also define $\theta_{j}^{\prime}(k)=\underline{\theta}$ for all $j \in \mathbf{N}$ and for all $k \in\{1, \ldots, n\}$. From the construction it follows that $\Delta(1) \theta_{j}(k)>\max \left\{\Delta(1) \theta_{1}, \Delta(1) \theta_{2}(k)\right\}$ for all $j \in \mathbf{N} /\{1,2\}$. Now consider profiles $\theta(S)=\left(\theta_{1}(S), \ldots, \theta_{n}(S)\right)$ where $\theta_{j}(S)=\theta_{j}$ if $j \notin S$ and $\theta_{j}(S)=\theta_{j}^{\prime}$ if $j \in S$. For all $s \in S$, the efficient queue position is behind all $j \notin S$, i.e. $\sigma_{s}^{*}(\theta(S)) \in\{n-|S|+1, \ldots, n\}$. This is because the queueing costs of all individuals $j \notin S$, in all queue positions strictly exceed the queueing costs of all individual $s \in S$. From the construction of $\left\{\theta_{3}, \ldots, \theta_{n}\right\}$ it follows that for all $\{j, l\} \in \mathbf{N} /\{1,2\}$ and for all $S \in \mathbf{N} /\{j, l\}$, with $j<l, \sigma_{j}^{*}\left(\theta_{j}, \theta_{l}, \theta_{-j-l}(S)\right)>\sigma_{l}^{*}\left(\theta_{j}, \theta_{l}, \theta_{-j-l}(S)\right)$. Observe that for all $S \in \mathbf{N} /\{1,2\}$, individuals 1 and 2 are immediate neighbours with any one of 1 and 2 having queue position $n-|S|$ and the other having queue position $n-|S|-1$. If, on the one hand, $\theta_{1}(n-|S|-1)+\theta_{2}(n-|S|) \leq$ $\theta_{1}(n-|S|)+\theta_{2}(n-|S|-1)$ then $\sigma_{1}^{*}\left(\theta_{1}, \theta_{2}, \theta_{-1-2}(S)\right)<\sigma_{2}^{*}\left(\theta_{1}, \theta_{2}, \theta_{-1-2}(S)\right)$. If, on the other hand, $\theta_{1}(n-|S|-1)+\theta_{2}(n-|S|) \geq \theta_{1}(n-|S|)+\theta_{2}(n-|S|-1)$ then $\sigma_{1}^{*}\left(\theta_{1}, \theta_{2}, \theta_{-1-2}(S)\right)>\sigma_{2}^{*}\left(\theta_{1}, \theta_{2}, \theta_{-1-2}(S)\right)$. Define, $Z(k, k+1)=\min \left\{\theta_{1}(k)+\right.$ $\left.\theta_{2}(k+1), \theta_{1}(k+1)+\theta_{2}(k)\right\}$ for all $k \in\{1, \ldots n-1\}$. Making use of the above observations and the definition of $Z(k, k+1)$ we get

$$
\sum_{S \subseteq \mathbf{N}}(-1)^{|S|} C(\theta(S))=\sum_{k=1}^{n-1}(-1)^{n-k-1}\left(\begin{array}{l}
n-2 \\
k-1
\end{array}\right)\left[Z(k, k+1)-\theta_{1}(k)-\theta_{2}(k)\right]
$$

For $\Omega$ to be FB implementable it is necessary from Lemma 2.1 that the RHS of equation (2.5) is zero for all $\left\{\theta_{1}, \theta_{2}\right\} \in \bar{\Theta}^{2}$ and satisfying CP. This crucially 
depends on the terms with $Z(k, k+1)$ in the RHS of equation (2.5). We claim that the RHS of (2.5) is zero if and only if either one of the two following conditions is satisfied then $\Omega$ is FB implementable.

1. $Z(k, k+1)=\theta_{1}(k)+\theta_{2}(k+1)$ for all $k \in\{1, \ldots, n-1\}$

2. $Z(k, k+1)=\theta_{1}(k+1)+\theta_{2}(k)$ for all $k \in\{1, \ldots, n-1\}$.

We first prove the if part of this claim. If condition (1) holds i.e. $Z(k, k+1)=$ $\theta_{1}(k)+\theta_{2}(k+1)$ for all $k \neq n$, then by substituting (1) in (2.5) and simplifying it we get

$$
\sum_{S \subseteq \mathbf{N}}(-1)^{|S|} C(\theta(S))=\sum_{k=1}^{n-1}(-1)^{n-k-1}\left(\begin{array}{l}
n-2 \\
k-1
\end{array}\right)\left\{\Delta(1) \theta_{2}(k)\right\}=0 .
$$

The last step follows from $\mathrm{CP}^{9}$. Similarly one can show that if condition (2) holds then

$$
\sum_{S \subseteq \mathbf{N}}(-1)^{|S|} C(\theta(S))=\sum_{k=1}^{n-1}(-1)^{n-k-1}\left(\begin{array}{l}
n-2 \\
k-1
\end{array}\right)\left\{\Delta(1) \theta_{1}(k)\right\}=0 .
$$

To prove the only if part of this claim we first assume that the claim is not true. This implies that there exists $\theta_{1}$ and $\theta_{2}$ for individuals 1 and 2 such that

(i) $\Delta(1) \theta_{1}(1) \geq \Delta(1) \theta_{2}(1)$

(ii) $\exists$ a set $T \subset\{2,3, \ldots, n-1\}$ such that $\Delta(1) \theta_{1}(r)<\Delta(1) \theta_{2}(r)$ for all $r \in T$ and

(iii) $\exists p \in\{1, \ldots, n-1\} / T$ such that $\Delta(1) \theta_{1}(p)>\Delta(1) \theta_{2}(p)$.

Using conditions $(i)-(i i)$ in $(2.5)$ and simplifying it we get

$$
\frac{\sum_{S \subseteq \mathbf{N}}(-1)^{|S|} C(\theta(S))=\sum_{r \in T}(-1)^{n-r-1}\left(\begin{array}{c}
n-2 \\
r-1
\end{array}\right)\left\{\Delta(1) \theta_{1}(r)-\Delta(1) \theta_{2}(r)\right\}}{{ }^{9} \text { If } \sum_{k=1}^{n}(-1)^{k-1}\left(\begin{array}{c}
n-1 \\
k-1
\end{array}\right) \theta_{j}(k)=0, \text { then } \sum_{k=1}^{n-1}(-1)^{k-1}\left(\begin{array}{c}
n-2 \\
k-1
\end{array}\right)\left\{\theta_{j}(k+1)-\theta_{j}(k)\right\}=0 .}
$$


From Lemma 2.1 we know that for FB implementability we need that the RHS of (2.6) must be zero. Now if $\Delta(1) \theta_{2}(k)=a>0$ for all $k \in\{1, \ldots, n-$ $1\}$ then construct a profile $\hat{\theta}_{1}$ such that $\Delta(1) \hat{\theta}_{1}(k)=(2 k+1) b$ with $b \in$ $\left(\frac{a}{2 n-3}, \frac{a}{2 n-1}\right)$. Applying the same type of construction i.e. $(2 n+1) b<\epsilon_{3}<$ $\ldots<\epsilon_{n}$ and $\underline{\theta} \in\left[0, \min \left\{\hat{\theta}_{1}, \theta_{2}\right\}\right]$ and defining $\theta_{j}$ for all $j \in\{3, \ldots, n\}$ and $\theta_{j}^{\prime}$ for all $j \in \mathbf{N}$ in the same way as before we get

$$
\sum_{S \subseteq \mathbf{N}}(-1)^{|S|} C(\theta(S))=\Delta(1) \theta_{2}(n-1)-\Delta(1) \hat{\theta}_{1}(n-1)=a-(2 n-1) b \neq 0 .
$$

If $\Delta(1) \theta_{2}(k)$ is not a constant for all $k \neq n$, then consider $\bar{\theta}_{2}$ such that $\bar{\theta}_{2}(k)=\theta_{2}(k)$ for all $k \in\{1, \ldots, n-2\}$ and select $\left\{\bar{\theta}_{2}(n-1), \bar{\theta}_{2}(n)\right\}$ in such a way that $\bar{a}=\Delta(1) \bar{\theta}_{2}(n-1)>\max \left\{\Delta(1) \bar{\theta}_{2}(k)\right\}_{k \neq n-1}$. Again, define $\bar{\theta}_{1}$ such that $\bar{\theta}_{1}(k)=k \epsilon_{1}$ where $\max \left\{\Delta(1) \bar{\theta}_{2}(k)\right\}_{k \neq n-1}<\epsilon_{1}<\bar{a}$. Again by applying the same type of construction we get

$$
\sum_{S \subseteq \mathbf{N}}(-1)^{|S|} C(\theta(S))=\Delta(1) \bar{\theta}_{2}(n-1)-\Delta(1) \bar{\theta}_{1}(n-1)=\bar{a}-\epsilon_{1} \neq 0 .
$$

Therefore, for a preference satisfying condition $(i)$ - (iii) and Lemma 2.1, we can find a preference in its neighbourhood that fails to satisfy Lemma 2.1. Thus, $\Omega$ satisfying CP is FB implementable only if either (1) holds or condition (2) holds. Since the selection of individuals 1 and 2 for the above construction was arbitrary, it follows that $\Omega$ satisfying $\mathrm{CP}$ is FB implementable only if for all $j \neq l$,

either $(a) \theta_{j}(k+1)-\theta_{j}(k) \geq \theta_{l}(k+1)-\theta_{l}(k)$ for all $k \neq n$

or $\quad(b) \theta_{j}(k+1)-\theta_{j}(k) \leq \theta_{l}(k+1)-\theta_{l}(k)$ for all $k \neq n$.

This condition means that the descending order of $\left\{\theta_{j}(2)-\theta_{j}(1)\right\}_{j=1}^{n}$ determines the efficient queue i.e. if $\theta_{j}(2)-\theta_{j}(1) \geq \theta_{l}(2)-\theta_{l}(1)$ in some state $\theta$, then $\sigma_{j}^{*}(\theta)<\sigma_{l}^{*}(\theta)$. Using Remark 2.3 we get $\Omega$ satisfies IP. The logic is quite simple, if for example, $\theta_{j}, \theta_{l}$ are such that $\theta_{j}(k+1)-\theta_{j}(k) \geq \theta_{l}(k+1)-\theta_{l}(k)$ for all $k \in\{1, \ldots, n-1\}$, then individual $j$ is served ahead of individual $l$ for all eliminations of $i \in \mathbf{N} /\{j, l\}$. This proves Step 2 .

Sufficiency: Consider the $\operatorname{sum} \sum_{l \neq j} h_{j}\left(\sigma_{j}^{*}\left(\theta_{-l}\right)\right)$ in state $\theta$ for individual $j \in \mathbf{N}$. From IP we get 
$\sum_{l \neq j} h_{j}\left(\sigma_{j}^{*}\left(\theta_{-l}\right)\right)$

$=\left(n-\sigma_{j}^{*}(\theta)\right) h_{j}\left(\sigma_{j}^{*}(\theta)\right)+\left(\sigma_{j}^{*}(\theta)-1\right) h_{j}\left(\sigma_{j}^{*}(\theta)-1\right)$

$=\theta_{j}\left(\sigma_{j}^{*}(\theta)\right)$ (from condition (2.3) in Proposition 2.1).

Now consider a particular Groves mechanism $\hat{\mathbf{M}}=\left\langle\sigma^{*}, \hat{t}\right\rangle$ where $\hat{\gamma}_{j}\left(\theta_{-j}\right)=$ $(n-1) \sum_{l \neq j} h_{l}\left(\sigma_{l}^{*}\left(\theta_{-j}\right)\right)$. Then it follows that

$\sum_{j \in \mathbf{N}} \hat{\gamma}_{j}\left(\theta_{-j}\right)$

$=(n-1) \sum_{j \in \mathbf{N}} \sum_{l \neq j} h_{l}\left(\sigma_{l}^{*}\left(\theta_{-j}\right)\right)$

$=(n-1) \sum_{j \in \mathbf{N}}\left\{\sum_{l \neq j} h_{j}\left(\sigma_{j}^{*}\left(\theta_{-l}\right)\right)\right\}$

$=(n-1) \sum_{j \in \mathbf{N}} \theta_{j}\left(\sigma_{j}^{*}(\theta)\right)$

$=(n-1) C(\theta)$.

This implies that for all $\theta \in \hat{\Theta}^{n}, \sum_{j \in \mathbf{N}} \hat{t}_{j}(\theta)=-(n-1) C(\theta)+\sum_{j \in \mathbf{N}} \hat{\gamma}_{j}\left(\theta_{-j}\right)=0$.

This section dealt with the restrictions required for FB implementability of the general queueing model. The next few sections restrict the cost of each individual to have a common functional form.

\section{Separable Cost Models}

In this section a class of queueing models, called separable cost queueing models, are considered. For separable cost queueing models, $\theta_{j}(k)$ satisfies the following conditions:

1. $\theta_{j}(k)=f(k) g\left(\theta_{j}\right)$ for all $j \in \mathbf{N}$, for all $k \in\{1,2, \ldots, n\}$ and for all $\theta_{j} \in \Theta$. Here $\Theta$ is assumed to be an interval in $\mathbf{R}_{+}$.

2. $g\left(\theta_{j}\right)>0$ for all $\theta_{j} \in \Theta$ and $g\left(\theta_{j}\right)$ is continuous and strictly increasing in $\theta_{j}$.

3. Finally, $f(k) \geq f(k-1)$ for all $k \in\{2, \ldots, n\}$. 
The first condition multiplicatively separates the cost of each individual for each position into two functions. The first function $f$ depends on the queue position. The second function $g$ depends on the type (or cost parameter i.e. $\theta_{j}$ ) of an individual. The second condition is a restriction on the type function. The third condition restricts the queueing cost function $f$ to be non-decreasing in queue positions. The second and third conditions together imply that $\theta_{j}(k+1) \geq \theta_{j}(k)$ for all $k \in\{1, \ldots, n-1\}$. The cost parameter (i.e. $\theta_{j}$ for $j \in \mathbf{N}$ ) is private information.

Each pair of functions $(f, g)$ together with $\mathbf{N}$ and type space $\Theta$, defines a separable cost queueing problem $\hat{\Omega}=\langle\mathbf{N}, \Theta,(f, g)\rangle$. A major benefit of such a specification is that the efficiency condition is completely transparent in this context. For a $\hat{\Omega}$, the queue $\sigma^{*}(\theta) \in \Sigma$ is efficient in state $\theta$ if for all $j \neq l, \theta_{j}>\theta_{l} \Rightarrow \sigma_{j}^{*}(\theta)<\sigma_{l}^{*}(\theta)$. Ties can be broken in many ways. A particular way of breaking ties, that will be followed in this paper, is to consider the natural ordering i.e. if $\theta_{j}=\theta_{j}$ and $j<l$ then $\sigma_{j}^{*}(\theta)<\sigma_{l}^{*}(\theta)^{10}$. The next Proposition is related to IP of $\hat{\Omega}$.

PROPOSITION $3.3 \hat{\Omega}$ satisfies IP.

PROOF: Consider $\hat{\Omega}=\langle\mathbf{N}, \Theta,(f, g)\rangle$ and an individual $j \in \mathbf{N}$ with queue position $\sigma_{j}^{*}(\theta)$ in state $\theta$. If $l \in P\left(\sigma^{*}(\theta), j\right)$ then $\sigma_{j}^{*}\left(\theta_{-l}\right)=\sigma_{j}^{*}(\theta)-1$ and $P\left(\sigma^{*}\left(\theta_{-l}\right), j\right)=P\left(\sigma^{*}(\theta), j\right) /\{l\}$ in $\hat{\Omega}_{N-l}=\langle\mathbf{N}-l, \Theta,(f, g)\rangle$. This is because individual $l$ is a predecessor of $j$ in $\hat{\Omega}=\langle\mathbf{N}, \Theta,(f, g)\rangle$. Also and because according to the definition of efficiency and the same tie breaking rule assumption, individual $j$ 's queue position with respect to all other individuals

\footnotetext{
${ }^{10}$ Note that the definition of efficient queue depends only on a pairwise comparison of individual types. In other words, if $\theta_{j}>\theta_{l}$, then for all $\theta_{-j-l} \in \Theta^{n-2}, \sigma_{j}^{*}(\theta)<\sigma_{l}^{*}(\theta)$. Also note that given the domain specification, there are states for which more than one ordering is efficient. So we have an efficiency correspondence for all such states. The tie breaking rule guarantees that in all states where more than one ordering is efficient, the decision picked is unique. Thus, a tie breaking rule guarantees a single valued selection of ordering decision from the efficiency correspondence.
} 
$\mathbf{N} /\{j, l\}$ remains unchanged in $\hat{\Omega}_{N-l}=\langle\mathbf{N}-l, \Theta,(f, g)\rangle$. Recall that the definition of efficiency for any separable cost queueing model $\hat{\Omega}$ depends only on the order of types of individuals. They remain invariant for the set of $n-1$ individuals included in any $\hat{\Omega}_{N-l}=\langle\mathbf{N} /\{l\}, \Theta,(f, g)\rangle$. If, on the other hand, $l \in \mathbf{N} /\left\{P\left(\sigma^{*}(\theta), j\right) \cup j\right\}$ then $\sigma_{j}^{*}\left(\theta_{-l}\right)=\sigma_{j}^{*}(\theta)$ and $P\left(\sigma^{*}\left(\theta_{-l}\right), j\right)=P\left(\sigma^{*}(\theta), j\right)$ in $\hat{\Omega}_{N-l}=\langle\mathbf{N}-l, \Theta,(f, g)\rangle$. This is because individual $l$ is a successor of individual $j$ in $\hat{\Omega}=\langle\mathbf{N}, \Theta,(f, g)\rangle$ and according to the definition of efficiency, individual $j$ 's queue position with respect to all other individuals $\mathbf{N} /\{j, l\}$ remains unchanged in $\hat{\Omega}_{N-l}=\langle\mathbf{N}-l, \Theta,(f, g)\rangle$.

The remainder of this section will deal with the question of FB implementability of the class of separable cost queueing model. The combinatorial property (or CP) is both necessary and sufficient for FB implementability of the class of separable cost queueing models. Note that $\hat{\Omega}=\langle\mathbf{N}, \Theta,(f, g)\rangle$ satisfies CP if

$$
\sum_{k=1}^{n}(-1)^{k-1}\left(\begin{array}{l}
n-1 \\
k-1
\end{array}\right) f(k)=0 .
$$

REMARK 3.4 From condition (2.3) it follows that $\hat{\Omega}$ satisfies CP, if and only if there exists a unique vector $H=\{h(1), \ldots, h(n-1)\}$ such that for all $k \in\{1, \ldots, n\}$,

$$
f(k)=(n-k) h(k)+(k-1) h(k-1)
$$

where $h(k)=\sum_{r=1}^{k}(-1)^{k-r} \frac{(k-1) !(n-k-1) !}{(r-1) !(n-r) !} f(r)$. The other observation that follows from condition (3.7) is

$$
\sum_{k=1}^{n} f(k)=n \sum_{k=1}^{n-1} h(k)
$$

Condition (3.8) will be useful in deriving later results.

PROPOSITION 3.4 $\hat{\Omega}=\langle\mathbf{N}, \Theta,(f, g)\rangle$ is FB implementable if and only if the cost function satisfies $\mathrm{CP}$. 
PROOF: To prove the necessary part of the Proposition we first construct two profiles and then apply Lemma 2.1. Let the two states $\theta$ and $\theta^{\prime}$ be of the following form: $\theta_{1}^{\prime}>\theta_{2}^{\prime}>\ldots>\theta_{n}^{\prime}>\theta_{1}>\theta_{2}>\ldots>\theta_{n}$. Now for all $S \subseteq \mathbf{N}$, we consider profiles $\theta(S)=\left(\theta_{1}(S), \ldots, \theta_{j}(S), \ldots, \theta_{n}(S)\right)$ where $\theta_{j}(S)=\theta_{j}$ if $j \notin S$ and $\theta_{j}(S)=\theta_{j}^{\prime}$ if $j \in S$.

For all $S \subseteq \mathbf{N} /\{1\}$ with profiles $\left(\theta_{1}, \theta_{-1}(S)\right), \sigma_{1}^{*}\left(\theta_{1}, \theta_{-1}(S)\right)=N-|S|$ and for all $S \subseteq \mathbf{N} /\{n\}$ with profiles $\left(\theta_{n}^{\prime}, \theta_{-n}(S)\right), \sigma_{n}^{*}\left(\theta_{n}^{\prime}, \theta_{-n}(S)\right)=|S|+1$. Therefore,

$\sum_{S \subseteq \mathbf{N} /\{1\}}(-1)^{|S|} C_{1}\left(\theta_{1}, \theta_{-1}(S)\right)=\sum_{|S|=0}^{n-1}(-1)^{|S|}\left(\begin{array}{c}n-1 \\ |S|\end{array}\right) f(n-|S|) g\left(\theta_{1}\right)$

and

$\sum_{S \subseteq \mathbf{N} /\{n\}}(-1)^{|S|} C_{n}\left(\theta_{n}^{\prime}, \theta_{-n}(S)\right)=\sum_{|S|=0}^{n-1}(-1)^{|S|}\left(\begin{array}{c}n-1 \\ |S|\end{array}\right) f(|S|+1) g\left(\theta_{n}^{\prime}\right)$.

For all $x_{j} \in\left\{\theta_{2}, \ldots, \theta_{n}, \theta_{1}^{\prime}, \ldots, \theta_{n-1}^{\prime}\right\}$, if the sets $\left\{m_{1}, \ldots, m_{p}\right\}$, all subsets of $S /\{j\}$, are such that $\sigma_{j}^{*}\left(x_{j}, \theta_{-j}\left(m_{q}\right)\right)=k$, for all $q \in\{1, \ldots, p\}$, then $\sum_{q=1}^{p}(-1)^{m_{q}}=0$. Therefore, $\sum_{S \subseteq \mathbf{N} /\{j\}}(-1)^{|S|} C\left(x_{j}, \theta_{-j}(S)\right)=0$.

Combining all these observations we get

$\sum_{S \subseteq \mathbf{N}}(-1)^{|S|} C(\theta(S))=\left\{g\left(\theta_{1}\right)-g\left(\theta_{n}^{\prime}\right)\right\} \sum_{k=1}^{n-1}(-1)^{k-1}\left(\begin{array}{l}n-1 \\ k-1\end{array}\right) f(k)$.

Applying Lemma 2.1 and using $g\left(\theta_{1}\right) \neq g\left(\theta_{n}^{\prime}\right)$ in the above equation we get

$$
\sum_{k=1}^{n-1}(-1)^{k-1}\left(\begin{array}{l}
n-1 \\
k-1
\end{array}\right) f(k)=0
$$

The sufficiency follows quite easily from Theorem 2.1.

Consider a queueing model $\hat{\Omega}=\langle\mathbf{N}, \Theta,(f, g)\rangle$. For convenience consider the "inverse" of the queue $\sigma^{*}$. That is, given $\theta \in \Theta^{n}$, let $\mu$ be a permutation such that $\theta_{\mu(1)} \geq \ldots \geq \theta_{\mu(n)}$. Furthermore, if $g\left(\theta_{j}\right)=g\left(\theta_{l}\right)$ and $j<l$, then $j \in P\left(\sigma^{*}(\theta), l\right)$. The explicit form of the transfer can be obtained from the following condition: 
$t_{\mu(k)}(\theta)=\sum_{q=1}^{k-1}\{(n-1) h(q)-f(q)\} g\left(\theta_{\mu(q)}\right)+\sum_{q=k+1}^{n}\{(n-1) h(q-1)-f(q)\} g\left(\theta_{\mu(q)}\right)$

where $h(q)=\sum_{r=1}^{q}(-1)^{q-r} \frac{(q-1) !(n-q-1) !}{(r-1) !(n-r) !} f(r)$ for all $q \in\{1, \ldots, n-1\}$.

The existence of FB implementable $\hat{\Omega}$ is already established in Mitra and Sen (1998) for $f(k)=k$. The question of the existence of other FB implementable separable cost queueing models is analysed in the next section.

\subsection{Applications}

In this section the existence of a fairly large class of FB implementable separable cost queueing models is established. We start by defining a broad class of queueing cost function.

DEFINITION $3.7 \tilde{f}_{a}^{n-2}$ is called a polynomial cost function of degree $n-2$ if

1. $\tilde{f}_{a}^{n-2}(k)=\sum_{p=1}^{n-2} a_{p} k^{p}$, for all $k \in\{1, \ldots, n\}$.

2. $\tilde{f}_{a}^{n-2}(k) \geq \tilde{f}_{a}^{n-2}(k-1)$, for all $k \in\{2, \ldots, n\}$.

It is important to observe that the class of polynomial cost depends crucially on the specification of the vector $a=\left(a_{1}, \ldots, a_{n-2}\right)$. Let $\tilde{\Omega}_{a}^{n-2}=$ $\left\langle\mathbf{N}, \Theta,\left(\tilde{f}_{a}^{n-2}, g\right)\right\rangle$ be a particular separable cost queueing model with polynomial cost $\tilde{f}_{a}^{n-2}$. Also let $\tilde{\Omega}^{n-2}$ be the class polynomial cost queueing models. Observe that from Proposition 3.3 it follows that all polynomial cost queueing models $\tilde{\Omega}_{a}^{n-2} \in \tilde{\Omega}^{n-2}$ satisfy IP. One can now introduce some special cases of the class of polynomial cost queueing models. One such special case is the factorial cost queueing model.

DEFINITION $3.8 f^{[m]}$ is called a factorial cost function of degree m if for all $m \in\{1, \ldots n-2\}$,

$$
f^{[m]}(k)=[k]_{m}=k(k-1) \ldots(k-m+1) .
$$


Here $m$ represents the queue position from which the $f^{[m]}$ is non-zero i.e.

$$
f^{[m]}(k)= \begin{cases}\frac{k !}{(k-m) !} & \text { if } k \geq m \\ 0 & \text { otherwise. }\end{cases}
$$

One can easily verify that for all $k \in\{2, \ldots, n\}$,

$$
f^{[m]}(k)-f^{[m]}(k-1)=\frac{m(k-1) !}{(k-m) !} \geq 0 .
$$

Let $\hat{\Omega}^{[m]}=\left\langle\mathbf{N}, \Theta,\left(f^{[m]}, g\right)\right\rangle$ be a separable cost queueing model with factorial cost of degree $m \leq n-2$.

Another type of polynomial cost queueing model is the standard cost queueing model.

DEFINITION $3.9 f^{m}$ is said to be a standard cost function of degree $\mathbf{m}$ if for all $m \in\{1, \ldots n-2\}, f^{m}(k)=k^{m}$.

One can easily that for all $k \in\{2, \ldots, n\}$,

$$
f^{m}(k)-f^{m}(k-1)=\left\{k^{m-1}+k^{m-2}(k-1)+\ldots+(k-1)^{m-1}\right\}>0 .
$$

Let $\hat{\Omega}^{m}=\left\langle\mathbf{N}, \Theta,\left(f^{m}, g\right)\right\rangle$ be a separable cost queueing model with standard queueing cost $f^{m}$ of degree $m \leq n-2$. Notice that $f^{[1]}=f^{1}$ i.e. factorial cost function of degree one and standard cost function of degree one are identical.

REMARK 3.5 Following remarks can be made about the polynomial cost $\tilde{f}_{a}^{n-2}$,

1. By selecting appropriate values of $a_{p}$ for all $p=1, \ldots, n-2$, one can get factorial cost of any degree $m \leq n-2$. For example, with $|\mathbf{N}|=4$, $a_{2}=1$ and $a_{1}=-1$ we get $\tilde{f}_{a}^{2}(k)=k^{2}-k=k(k-1)=f^{[2]}(k)$. In general, $\tilde{f}_{a}^{n-2}$ is a factorial cost of degree $m \leq n-2$ if $a_{p}=s(m, p)$ for all $p=1, \ldots, m$ and $a_{p}=0$ otherwise. $s(m, p)$ for all $p=1, \ldots, m$, are Stirling number of the first kind ${ }^{11}$.

\footnotetext{
${ }^{11} \mathrm{~A}$ Stirling number of the first kind, $s(m, p)$, is defined as the coefficient of $x^{p}$ in the expansion of $[x]_{p}=x(x-1) \ldots(x-p+1)$, i.e. $[x]_{p}=\sum_{p=1}^{m} s(m, p) x^{p}$. For further references see Tomescu and Melter (1985).
} 
2. A polynomial cost $\tilde{f}_{a}^{n-2}$ is a standard cost of degree $m$ if $a_{m}=1$ and $a_{p}=0$ for all $p \neq m$.

THEOREM 3.2 $\tilde{\Omega}_{a}^{n-2} \in \tilde{\Omega}^{n-2}$ is FB implementable.

We state and prove two Lemma that will be used in proving Theorem 3.2 .

LEMMA 3.2 $\hat{\Omega}^{[m]}$ is FB implementable.

PROOF: To prove this Lemma we will have to show that $\hat{\Omega}^{[m]}$ satisfies CP. From the definition of $f^{[m]}$, it follows that

$$
\begin{aligned}
& \sum_{k=1}^{n}(-1)^{k-1}\left(\begin{array}{l}
n-1 \\
k-1
\end{array}\right) f^{[m]}(k) \\
= & \sum_{k=1}^{n}(-1)^{k-1}\left(\begin{array}{c}
n-1 \\
k-1
\end{array}\right) \frac{k !}{(k-m) !} \\
= & {[n-1]_{m-1}(-1)^{m-1} \sum_{k=m}^{n}(-1)^{k-m}\left(\begin{array}{c}
n-m \\
k-m
\end{array}\right)\{(k-m)+m\} } \\
= & {[n-1]_{m}(-1)^{m} \sum_{k=m+1}^{n}(-1)^{k-m-1}\left(\begin{array}{c}
n-m-1 \\
k-m-1
\end{array}\right) } \\
& +m[n-1]_{m-1}(-1)^{m-1} \sum_{k=m}^{n}(-1)^{k-m}\left(\begin{array}{c}
n-m \\
k-m
\end{array}\right) \\
= & 0 .
\end{aligned}
$$

LEMMA $3.3 \hat{\Omega}^{m}$ is FB implementable.

PROOF: To prove this Lemma we use the following mathematical identity

$$
k^{m}=\sum_{q=1}^{m} S(m, q)[k]_{q}
$$

where $[k]_{q}=k(k-1) \ldots(k-q+1)$ and $S(m, q)$ are Stirling number of the second kind ${ }^{12}$.

\footnotetext{
${ }^{12} \mathrm{~A}$ Stirling number of the second kind $S(m, q)$, is defined as the coefficient of $[x]_{q}$ in the expansion of $x^{q}$, i.e., $x^{q}=\sum_{q=0}^{m} S(m, q)[x]_{q}$. Stirling number of the second kind are such that $S(m, 1)=S(m, m)=1$. Moreover, these numbers are unimodal i.e. they satisfy one
} 
From (3.11), it follows that

$$
\begin{aligned}
& \sum_{k=1}^{n}(-1)^{k-1}\left(\begin{array}{l}
n-1 \\
k-1
\end{array}\right) f^{m}(k) \\
= & \sum_{k=1}^{n}(-1)^{k-1}\left(\begin{array}{l}
n-1 \\
k-1
\end{array}\right) \sum_{q=1}^{m} S(m, q) f^{[q]}(k) \\
= & \sum_{q=1}^{m} S(m, q)\left\{\sum_{k=1}^{n}(-1)^{k-1}\left(\begin{array}{l}
n-1 \\
k-1
\end{array}\right) f^{[q]}(k)\right\} \\
= & 0 \text { (from Lemma 3.2). }
\end{aligned}
$$

PROOF OF THEOREM 3.2: To prove this Theorem we will have to show that $\tilde{\Omega}_{a}^{n-2}$ satisfies CP. Given the form of the cost function we get

$$
\begin{aligned}
& \sum_{k=1}^{n}(-1)^{k-1}\left(\begin{array}{l}
n-1 \\
k-1
\end{array}\right) \tilde{f}_{a}^{n-2}(k) \\
= & \sum_{k=1}^{n}(-1)^{k-1}\left(\begin{array}{l}
n-1 \\
k-1
\end{array}\right)\left\{\sum_{p=1}^{n-2} a_{p} f^{p}(k)\right\} \\
= & \sum_{p=1}^{n-2} a_{p}\left\{\sum_{k=1}^{n}(-1)^{k-1}\left(\begin{array}{l}
n-1 \\
k-1
\end{array}\right) f^{p}(k)\right\} \\
= & 0 \text { (from Lemma 3.3). }
\end{aligned}
$$

The remaining part of this section deals with examples of different polynomial cost queueing models with $|\mathbf{N}|=4$. The first two examples are factorial cost queueing models of degree one and two. The third example is a standard cost queueing model of degree two. The final example is a polynomial cost queueing model of degree two.

EXAMPLE 3.2 Consider $\hat{\Omega}^{[1]}=\left\langle\mathbf{N}=\{1,2,3,4\}, \Theta,\left(f^{[1]}, g\right)\right\rangle$ where the queueing cost function is of the form $f^{[1]}(k)=k$, for all $k=1,2,3,4$.

of the following formulae:

1. $1=S(m, 1)<S(m, 2)<\ldots<S(m, M(m))>S(m, M(m)-1) \ldots>S(m, m)=1$ or

2. $1=S(m, 1)<S(m, 2)<\ldots<S(m, M(m)-1)=S(m, M(m))>\ldots>S(m, m)=$ 1

and $M(m+1)=M(m)$ or $M(m+1)=M(m)+1$ where $M(m)=\max \{q \mid S(m, q)$ is maximum; $1 \leq q \leq m\}$. For a better understanding see Tomescu and Melter (1985). 
Condition (3.7) gives $h^{[1]}(k)=\sum_{r=1}^{k}(-1)^{k-r} \frac{(k-1) !(n-k-1) !}{(r-1) !(n-r) !} f^{[1]}(r)$. Elementary computation gives $\mathbf{H}^{[1]}=\left\{h^{[1]}(1)=\frac{1}{3}, h^{[1]}(2)=\frac{5}{6}, h^{[1]}(3)=\frac{4}{3}\right\}$.

Now consider a state $\theta=\left(\theta_{1}, \theta_{2}, \theta_{3}, \theta_{4}\right)$ such that $\theta_{1} \geq \theta_{2} \geq \theta_{3} \geq \theta_{4}$. This means that $\sigma_{j}^{*}(\theta)=j$, for all $j=1,2,3,4$. We can see that for all $j \neq l$,

$$
\sigma_{j}^{*}\left(\theta_{-l}\right)= \begin{cases}j & \text { if } \sigma_{j}^{*}(\theta)<\sigma_{l}^{*}(\theta) \\ j-1 & \text { if } \sigma_{j}^{*}(\theta)>\sigma_{l}^{*}(\theta) .\end{cases}
$$

Consider the Groves transfer, as defined in condition (3.10). Computation of the transfers give

$$
\begin{aligned}
t_{1}(\theta)= & -\left\{f^{[1]}(2) g\left(\theta_{2}\right)+f^{[1]}(3) g\left(\theta_{3}\right)+f^{[1]}(4) g\left(\theta_{4}\right)\right\} \\
& +(n-1)\left\{h^{[1]}(1) g\left(\theta_{2}\right)+h^{[1]}(2) g\left(\theta_{3}\right)+h^{[1]}(3) g\left(\theta_{4}\right)\right\} \\
= & -g\left(\theta_{2}\right)-\frac{1}{2} g\left(\theta_{3}\right), \\
t_{2}(\theta)= & -\left\{f^{[1]}(1) g\left(\theta_{1}\right)+f^{[1]}(3) g\left(\theta_{3}\right)+f^{[1]}(4) g\left(\theta_{4}\right)\right\} \\
& +(n-1)\left\{h^{[1]}(1) g\left(\theta_{1}\right)+h^{[1]}(2) g\left(\theta_{3}\right)+h^{[1]}(3) g\left(\theta_{4}\right)\right\} \\
= & -\frac{1}{2} g\left(\theta_{3}\right), \\
t_{3}(\theta)= & -\left\{f^{[1]}(1) g\left(\theta_{1}\right)+f^{[1]}(2) g\left(\theta_{2}\right)+f^{[1]}(4) g\left(\theta_{4}\right)\right\} \\
& +(n-1)\left\{h^{[1]}(1) g\left(\theta_{1}\right)+h^{[1]}(2) g\left(\theta_{2}\right)+h^{[1]}(3) g\left(\theta_{4}\right)\right\} \\
= & \frac{1}{2} g\left(\theta_{2}\right), \\
t_{4}(\theta)= & -\left\{f^{[1]}(1) g\left(\theta_{1}\right)+f^{[1]}(2) g\left(\theta_{2}\right)+f^{[1]}(3) g\left(\theta_{3}\right)\right\} \\
& +(n-1)\left\{h^{[1]}(1) g\left(\theta_{1}\right)+h^{[1]}(2) g\left(\theta_{2}\right)+h^{[1]}(3) g\left(\theta_{3}\right)\right\} \\
= & \frac{1}{2} g\left(\theta_{2}\right)+g\left(\theta_{3}\right) .
\end{aligned}
$$

Note that $\sum_{j=1}^{4} t_{j}(\theta)=0$. To write an explicit form of the transfers for each state $\theta \in \Theta$, consider the "inverse" of the order $\sigma^{*}$, suppose $\mu$ is a permutation such that

$$
\theta_{\mu(1)} \geq \theta_{\mu(2)} \geq \theta_{\mu(3)} \geq \theta_{\mu(4)}
$$

The transfers are

$t_{\mu(1)}(\theta)=-g\left(\theta_{\mu(2)}\right)-\frac{1}{2} g\left(\theta_{\mu(3)}\right)$, 
$t_{\mu(2)}(\theta)=-\frac{1}{2} g\left(\theta_{\mu(3)}\right)$,

$t_{\mu(3)}(\theta)=\frac{1}{2} g\left(\theta_{\mu(2)}\right)$ and

$t_{\mu(4)}(\theta)=\frac{1}{2} g\left(\theta_{\mu(2)}\right)+g\left(\theta_{\mu(3)}\right)$. Observe that $\sum_{k=1}^{4} t_{\mu(k)}(\theta)=0$.

The reason why these transfers are incentive compatible is intuitive. The individuals with first and second positions in the queue compensates the individuals with third and last positions in the queue in such a way that truth-telling is a dominant strategy for all the individuals. The amount that the first individual pays exceeds the amount paid by the second individual by $g\left(\theta_{\mu(2)}\right)$. So, by moving in the second position the first individual with type $\theta_{\mu(1)}$ cannot benefit because his reduction in payment will be $g\left(\theta_{\mu(2)}\right)$ and his increase in queueing cost will be $g\left(\theta_{\mu(1)}\right)\left(>g\left(\theta_{\mu(2)}\right)\right)$. Similarly, the individual having second position in the queue cannot benefit by moving ahead in the queue. In which case, he will have to pay $g\left(\theta_{\mu(1)}\right)$ more and his reduction in cost will be $g\left(\theta_{\mu(2)}\right)\left(<g\left(\theta_{\mu(1)}\right)\right)$. One can, by applying similar arguments, check that these transfers are dominant strategy incentive compatible for all individuals.

EXAMPLE 3.3 Consider $\hat{\Omega}^{[2]}=\left\langle\mathbf{N}=\{1,2,3,4\}, \Theta,\left(f^{[2]}, g\right)\right\rangle$ where the queueing cost function is of the form $f^{[2]}(k)=k(k-1)$, for all $k=1,2,3,4$. Using condition (3.7) we get $h^{[2]}(r)=\sum_{r=1}^{m}(-1)^{m-r} \frac{(m-1) !(n-m-1) !}{(r-1) !(n-r) !} f^{[2]}(r)$.

Elementary computation gives $\mathbf{H}^{[2]}=\left\{h^{[2]}(1)=0, h^{[2]}(2)=1, h^{[2]}(3)=4\right\}$.

Consider a state $\theta \in \Theta^{4}$ and $\theta_{\mu(1)} \geq \theta_{\mu(2)} \geq \theta_{\mu(3)} \geq \theta_{\mu(4)}{ }^{13}$. The transfers are:

$t_{\mu(1)}(\theta)=-2 g\left(\theta_{\mu(2)}\right)-3 g\left(\theta_{\mu(3)}\right)$,

$t_{\mu(2)}(\theta)=-3 g\left(\theta_{\mu(3)}\right)$,

$t_{\mu(3)}(\theta)=g\left(\theta_{\mu(2)}\right)$ and

$t_{\mu(4)}(\theta)=g\left(\theta_{\mu(2)}\right)+6 g\left(\theta_{\mu(3)}\right)$. Adding the transfers give $\sum_{k=1}^{4} t_{\mu(k)}(\theta)=0$. By considering deviations one can find that truth-telling is a dominant strategy

\footnotetext{
${ }^{13} \mu$ is the inverse of $\sigma^{*}$ as defined in the previous example.
} 
for all individuals.

Observe that the factorial cost of degree 1 is of the same form as the standard cost of degree 1, i.e. $f^{[1]}(k)=f^{1}(k)=k$. An example of a standard cost queueing model for $|\mathbf{N}|=4$ with $m=2$ is given below.

EXAMPLE 3.4 Consider $\hat{\Omega}^{2}=\left\langle\mathbf{N}=\{1,2,3,4\}, \Theta,\left(f^{2}, g\right)\right\rangle$ where the queueing cost function is of the form $f^{2}(k)=k^{2}$, for all $k=1,2,3,4$. Observe that from Proposition 3.3 it follows that

$f^{2}(k)=k^{2}=S(2,1) k+S(2,2) k(k-1)$,

where $\{S(2,2), S(2,1)\}$ are Stirling numbers of the second kind satisfying $S(2,1)=S(2,2)=1^{14}$.

From condition (3.7) we know that $h^{2}(r)=\sum_{r=1}^{m}(-1)^{m-r} \frac{(m-1) !(n-m-1) !}{(r-1) !(n-r) !} f^{2}(r)$. By substituting the factorial cost representation as explained above we observe that

$h^{2}(r)=h^{[1]}(r)+h^{[2]}(r)$. Thus, $\mathbf{H}^{2}=\left\{h^{2}(1)=\frac{1}{3}, h^{2}(2)=\frac{11}{6}, h^{2}(3)=\frac{16}{3}\right\}$.

For a state $\theta \in \Theta^{4}$ with $\theta_{\mu(1)} \geq \theta_{\mu(2)} \geq \theta_{\mu(3)} \geq \theta_{\mu(4)}$, the explicit form of the transfers are:

$t_{\mu(1)}(\theta)=-3 g\left(\theta_{\mu(2)}\right)-\frac{7}{2} g\left(\theta_{\mu(3)}\right)$,

$t_{\mu(2)}(\theta)=-\frac{7}{2} g\left(\theta_{\mu(3)}\right)$,

$t_{\mu(3)}(\theta)=\frac{3}{2} g\left(\theta_{\mu(2)}\right)$ and

$t_{\mu(4)}(\theta)=\frac{3}{2} g\left(\theta_{\mu(2)}\right)+7 g\left(\theta_{\mu(3)}\right)$. Check that $\sum_{k=1}^{4} t_{\mu(k)}(\theta)=0$. With these transfers it is clear that the individuals getting first and second positions in the queue compensate the individuals getting third and fourth positions in the queue in such a way that truth-telling is a dominant strategy for all individuals.

\footnotetext{
${ }^{14}$ For $m=3, f^{3}(k)=k^{3}=S(3,1) k+S(3,2) k(k-1)+S(3,3) k(k-1)(k-2)$ where $S(3,1)=S(3,3)=1$ and $S(3,2)=3$. For other $m s^{\prime}$ one can similarly represent standard cost as a weighted sum of factorial costs of degrees $\{1, \ldots, m\}$ where the weights are Stirling numbers of the second kind.
} 
EXAMPLE 3.5 Consider $\tilde{\Omega}_{a}^{2}=\left\langle\mathbf{N}=\{1,2,3,4\}, \Theta,\left(\tilde{f}_{a}^{2}, g\right)\right\rangle$ where the queueing cost function is of the form $\tilde{f}_{a}^{2}(k)=a_{1} k+a_{2} k^{2}$, for all $k=1,2,3,4^{15}$. Observe that

$$
\begin{aligned}
& \tilde{f}_{a}^{2}(k) \\
= & a_{1} f^{1}(k)+a_{2} f^{2}(k) \\
= & a_{1} f^{[1]}(k)+a_{2}\left\{S(2,1) f^{[1]}(k)+S(2,2) f^{[2]}(k)\right\} \\
= & \left\{a_{1}+a_{2}\right\} f^{[1]}(k)+a_{2} f^{[2]}(k) .
\end{aligned}
$$

From condition (3.7) we know that $\tilde{h}_{a}^{2}(k)=\sum_{r=1}^{k}(-1)^{k-r} \frac{(k-1) !(n-k-1) !}{(r-1) !(n-r) !} \tilde{f}_{a}^{2}(r)$.

By substituting the factorial cost representation it is quite easy to observe that $\tilde{h}_{a}^{2}(k)=\left\{a_{1}+a_{2}\right\} h^{[1]}(k)+a_{2} h^{[2]}(k)$ for all $k=1,2,3$.

Thus $\tilde{\mathbf{H}}_{a}^{2}=\left\{\tilde{h}_{a}^{2}(1)=\frac{1}{3}\left(a_{1}+a_{2}\right), \tilde{h}_{a}^{2}(2)=\frac{5}{6} a_{1}+\frac{11}{6} a_{2}, \tilde{h}_{a}^{2}(3)=\frac{4}{3} a_{1}+\frac{16}{3} a_{2}\right\}$.

Consider a state $\theta \in \Theta^{4}$ and $\theta_{\mu(1)} \geq \theta_{\mu(2)} \geq \theta_{\mu(3)} \geq \theta_{\mu(4)}$. Here the transfers are:

$$
\begin{aligned}
& t_{\mu(1)}(\theta)=-\left(a_{1}+3 a_{2}\right) g\left(\theta_{\mu(2)}\right)-\frac{1}{2}\left(a_{1}+7 a_{2}\right) g\left(\theta_{\mu(3)}\right), \\
& t_{\mu(2)}(\theta)=-\frac{1}{2}\left(a_{1}+7 a_{2}\right) g\left(\theta_{\mu(3)}\right) \\
& t_{\mu(3)}(\theta)=\frac{1}{2}\left(a_{1}+3 a_{2}\right) g\left(\theta_{\mu(2)}\right) \text { and } \\
& t_{\mu(4)}(\theta)=\frac{1}{2}\left(a_{1}+3 a_{2}\right) g\left(\theta_{\mu(2)}\right)+\left(a_{1}+7 a_{2}\right) g\left(\theta_{\mu(3)}\right) .
\end{aligned}
$$

Adding up the transfers for all $k=\{1,2,3,4\}$ gives $\sum_{k=1}^{4} t_{\mu(k)}(\theta)=0$.

The analysis of the class of separable cost queueing models in this section suggests the existence of a fairly large class of FB implementable separable

\footnotetext{
${ }^{15}$ Note that $\tilde{\Omega}_{a}^{2}=\left\langle\mathbf{N}=\{1,2,3,4\}, \Theta,\left(\tilde{f}_{a}^{2}, g\right)\right\rangle$ is a polynomial cost queueing model if $\tilde{f}_{a}^{2}(k+1)-\tilde{f}_{a}^{2}(k) \geq 0$ for all $k=1,2,3$. Therefore one of the following conditions must satisfied.

1. $a_{1}<0 \Rightarrow a_{1}+3 a_{2} \geq 0$

2. $a_{1}=0 \Rightarrow a_{2} \geq 0$ and

3. $a_{1}>0 \Rightarrow a_{1}+7 a_{2} \geq 0$.
} 
cost queueing models. This FB implementable class increases with the number of individuals. For example, factorial cost and standard cost queueing models are of degree $m \leq n-2$. The class of polynomial cost queueing model are also of degree $n-2$. So degree of $n-2$ plays an important role in FB implementability of queueing models. This is because $\mathrm{CP}$ requires that the $(n-1)$ th order difference at queue position 1 must be zero. Thus polynomial costs of degree more than $n-2$ are not FB implementable.

\section{A General Class}

A more general class of queueing models that are FB implementable is considered in this section. This class is defined by the following property.

DEFINITION 4.10 $\Omega$ satisfies Property $\mathbf{G}$ if for all $j \in \mathbf{N}$ and for all $k \in\{1, \ldots, n\}, \theta_{j}(k)$ satisfies the following conditions:

1. $\theta_{j}(k)=\sum_{p=1}^{M} f^{p}(k) g^{p}\left(\theta_{j}\right)+\beta_{j}\left(\theta_{j}\right)$ for all $j \in \mathbf{N}$, for all $k \in\{1, \ldots, n\}$, for all $\theta_{j} \in \Theta$ and for all $p \in\{1, \ldots, M\}$.

2. For all $p \in\{1, \ldots, M\}, g^{p}\left(\theta_{j}\right)>0$ for all $\theta_{j} \in \Theta$ and $g^{p}\left(\theta_{j}\right)$ is continuous and strictly increasing in $\theta_{j}$.

3. For all $p \in\{1, \ldots, M\}, f^{p}(k) \geq f^{p}(k-1)$ for all $k \in\{2, \ldots, n\}$ and $\sum_{k=1}^{n}(-1)^{k-1}\left(\begin{array}{l}n-1 \\ k-1\end{array}\right) f^{p}(k)=0$.

Let $\Omega^{G}$ be the class of queueing models satisfying Property $\mathbf{G}$. Observe that a queueing model $\Omega^{G} \in \Omega^{G}$ with $M=1$ and $\beta_{j}\left(\theta_{j}\right)=0$ for all $\theta_{j} \in \Theta$ and for all $j \in \mathbf{N}$, is a first best implementable separable cost queueing model. In the next result it is proved that this class of queueing models is FB implementable.

THEOREM 4.3 $\Omega^{G} \in \Omega^{G}$ is FB implementable. 
PROOF: To prove this Theorem we first argue that $\Omega^{G} \in \Omega^{G}$ satisfies IP. This follows from the fact that the efficient queue in any $\Omega^{G} \in \Omega^{G}$ depends on the ordering of the types as was the case for separable cost queueing models. Hence by following same arguments as in Proposition 3.3, one can prove that $\Omega^{G} \in \Omega^{G}$ satisfies IP.

The next step is to specify a Groves transfer and show that $\Omega^{G} \in \Omega^{G}$ is balanced for all states $\theta \in \Theta^{n}$. Observe that, for all $p \in\{1, \ldots, M\}$, $\sum_{k=1}^{n}(-1)^{k-1}\left(\begin{array}{l}n-1 \\ k-1\end{array}\right) f^{p}(k)=0$ implies from condition (3.7) that for all $p \in$ $\{1, \ldots, M\}$, there exists a unique vector $\mathbf{H}^{p}=\left\{h^{p}(1), \ldots, h^{p}(n-1)\right\}$ such that for all $k \in\{1, \ldots, n\}, f^{p}(k)=(n-k) h^{p}(k)+(k-1) h^{p}(k-1)$. Given $\Omega^{G} \in \Omega^{G}$ satisfy IP, by following the sufficiency argument in Proposition 3.4 we get that for all $\theta \in \Theta^{n}$ and for all $p \in\{1, \ldots, M\}, f^{p}\left(\sigma_{j}^{*}(\theta)\right)=$ $\sum_{l \neq j} h^{p}\left(\sigma_{j}^{*}\left(\theta_{-l}\right)\right)$.

Now consider a particular Groves mechanism $\hat{\mathbf{M}} \equiv\left\langle\sigma^{*}, t\right\rangle$ where

$$
\hat{\gamma}_{j}\left(\theta_{-j}\right)=\sum_{l \neq j}\left\{\beta_{l}\left(\theta_{l}\right)+(n-1) \sum_{p=1}^{M} g^{p}\left(\theta_{l}\right) h^{p}\left(\sigma_{l}^{*}\left(\theta_{-j}\right)\right)\right\}
$$

Then it follows that

$$
\begin{aligned}
& \sum_{j \in \mathbf{N}} \hat{\gamma}_{j}\left(\theta_{-j}\right) \\
& =(n-1) \sum_{j \in \mathbf{N}} \sum_{p=1}^{M} \sum_{l \neq j} g^{p}\left(\theta_{l}\right) h^{p}\left(\sigma_{l}^{*}\left(\theta_{-j}\right)\right)+(n-1) \sum_{j \in \mathbf{N}} \beta_{j}\left(\theta_{j}\right) \\
& =(n-1) \sum_{j \in \mathbf{N}} \sum_{p=1}^{M} g^{p}\left(\theta_{j}\right)\left\{\sum_{l \neq j} h^{p}\left(\sigma_{j}^{*}\left(\theta_{-l}\right)\right)\right\}+(n-1) \sum_{j \in \mathbf{N}} \beta_{j}\left(\theta_{j}\right) \\
& =(n-1) \sum_{j \in \mathbf{N}}\left\{\sum_{p=1}^{M} f^{p}\left(\sigma_{j}^{*}(\theta)\right) g^{p}\left(\theta_{j}\right)+\beta_{j}\left(\theta_{j}\right)\right\} . \\
& =(n-1) C(\theta) .
\end{aligned}
$$

This implies that for all $\theta \in \hat{\Theta}^{n}, \sum_{j \in \mathbf{N}} t_{j}(\theta)=-(n-1) C(\theta)+\sum_{j \in \mathbf{N}} \hat{\gamma}_{j}\left(\theta_{-j}\right)=0$.

One can easily verify the following results:

- $\Omega^{G} \in \Omega^{G}$ satisfies CP. 
- The class of FB implementable separable cost queueing models is a special case of $\Omega^{G}$.

\section{A Discounted Cost Model}

In all the previous sections, the queueing models that were considered had a cost specification that was increasing over time. Discounting is another standard way of evaluating costs or benefits which accrue over time. For example, in repeated games, one way to analyse benefits of an individual over time is to study the discounted payoff of the individual. Similarly, in some bargaining models, the cost of delay is measured in terms of a constant discount rate. One can think of many other situations where discounting is a standard way of measuring the cost of delay. Therefore, another way of specifying costs in a queueing model is to consider a decrease in gross benefit from the service over time. The general model specified in section two is general enough to include this model as a special case in the following way.

DEFINITION 5.11 A queueing model $\Omega^{d}=\langle\mathbf{N},[0,1]\rangle$ is called a discounted cost model if for all $j \in \mathbf{N}$ and for all $k \in\{1, \ldots, n\}, \theta_{j}(k)=$ $\left(1-\theta_{j}^{k}\right) v_{j}$ where $\theta_{j} \in[0,1]$.

The utility of an individual $j$ in $\Omega^{d}$ is of the form $U_{j}\left(k, t_{j} ; \theta_{j}\right)=\theta_{j}^{k} v_{j}+t_{j}$. This form of the utility is obtained by substituting $\theta_{j}(k)=\left(1-\theta_{j}^{k}\right) v_{j}$ in the general queueing model. Here $\theta_{j} \in[0,1]$ represents the type of individual $j$ which is private information. One can check that $\theta_{j}(k+1)-\theta_{j}(k)=\theta_{j}^{k}\left(1-\theta_{j}\right) v_{j} \geq 0$ for all $k \in\{1, \ldots, n-1\}$.

It is quite easy to observe that for $\Omega^{d}$, the domain specified satisfies Holmström's definition of "convex domains" and hence can be implemented only by Groves mechanism. For discounted cost queueing models, CP means that for all $j \in \mathbf{N}, \theta_{j}\left(1-\theta_{j}\right)^{n}=0$ i.e. $\theta_{j} \in\{0,1\}$. Thus for all $\theta_{j} \in(0,1)$ and for all $j \in \mathbf{N}, \mathrm{CP}$ is not satisfied. The next Proposition looks at the question of FB implementability of $\Omega^{d}$. 
PROPOSITION 5.5 $\Omega^{d}=\langle\mathbf{N},[0,1]\rangle$ is not FB implementable.

PROOF: To prove this Proposition we will consider two states and apply Lemma 2.1. Consider a particular individual $m \in \mathbf{N}$ such that $v_{m} \leq v_{j}$ for all $j \in \mathbf{N} /\{m\}$. Let $\theta_{m}=\frac{1}{3}, \theta_{j}=\frac{1}{2}$ for all $j \in \mathbf{N} /\{m\}$ and $\bar{\theta}_{j}=0$ for all $j \in \mathbf{N}$. Consider two states $\theta=\left(\theta_{1}, \ldots, \theta_{n}\right)$ and $\bar{\theta}=\left(\bar{\theta}_{1}, \ldots, \bar{\theta}_{n}\right)$. Elementary calculation yields $\theta_{m}^{k}\left(1-\theta_{m}\right) v_{m}<\theta_{j}^{k}\left(1-\theta_{j}\right) v_{j}$ for all $k \in\{1, \ldots, n\}$ and for all $j \in \mathbf{N} /\{m\}$. Therefore, $n=\sigma_{m}^{*}(\theta)>\sigma_{j}^{*}(\theta)$ for all $j \in \mathbf{N} /\{m\}$. Now for all $S \subseteq \mathbf{N}$, we consider profiles $\theta(S)=\left(\theta_{1}(S), \ldots, \theta_{j}(S), \ldots, \theta_{n}(S)\right)$ where $\theta_{j}(S)=\theta_{j}$ if $j \notin S$ and $\theta_{j}(S)=\bar{\theta}_{j}=0$ if $j \in S$. For all $S \subseteq \mathbf{N} /\{m\}$ with profiles $\left(\theta_{m}, \theta_{-m}(S)\right), \sigma_{m}^{*}\left(\theta_{m}, \theta_{-m}(S)\right)=n-|S|$. Therefore,

$\sum_{S \subseteq \mathbf{N} /\{m\}}(-1)^{|S|} C_{m}\left(\theta_{m}, \theta_{-m}(S)\right)=\sum_{|S|=0}^{n-1}(-1)^{|S|}\left(\begin{array}{c}n-1 \\ |S|\end{array}\right) \theta_{m}^{(n-|S|)} v_{m}$

For all $x_{j} \in\left\{\theta_{2}, \ldots, \theta_{n}\right\} /\left\{\theta_{m}\right\}$, if the sets $\left\{m_{1}, \ldots, m_{p}\right\}$, all subsets of $S \subseteq \mathbf{N} /\{j\}$, are such that $\sigma_{j}^{*}\left(x_{j}, \theta_{-j}\left(m_{q}\right)\right)=k$, for all $q \in\{1, \ldots, p\}$, then $\sum_{q=1}^{p}(-1)^{m_{q}}=0$ for all $k$. Therefore, $\sum_{S \subseteq \mathbf{N} /\{j\}}(-1)^{|S|} C\left(x_{j}, \theta_{-j}(S)\right)=0$.

Combining all these observations and the fact that $\bar{\theta}_{j}=0$ for all $j \in \mathbf{N}$ we get

$\sum_{S \subseteq \mathbf{N}}(-1)^{|S|} C(\theta(S))$

$=\sum_{k=1}^{n}(-1)^{k-1}\left(\begin{array}{l}n-1 \\ k-1\end{array}\right) \theta_{m}^{n-k+1}$

$=\theta_{m}\left(\theta_{m}-1\right)^{n}=\frac{1}{3}\left(\frac{1}{3}-1\right)^{n} \neq 0$.

From the constructions in the previous Proposition it is easy to see that if $\theta$ is such that $\theta_{j} \in\{0,1\}$ for all $j \in \mathbf{N}$, then $\Omega^{d}$ is FB implementable. Therefore discounted cost queueing model cannot be FB implemented simply because it fails to satisfy $\mathrm{CP}$. This result confirms the importance of $\mathrm{CP}$ as a necessary condition for FB implementability of any queueing model. 


\section{Individual Rationality}

This section deals with the identification of the sub-class of individually rational queueing models within the class of FB implementable queueing models discussed earlier.

DEFINITION 6.12 A mechanism $\mathbf{M} \equiv\langle\sigma, \mathbf{t}\rangle$ is individually rational for a decision problem $\Omega$, if for all $j \in \mathbf{N}$ and for all $\theta \in \Theta^{N}$,

$$
U_{j}\left(d_{j}(\theta), t_{j}(\theta), \theta_{j}\right) \geq 0
$$

This definition means that each individual has an outside option independent of his valuation which gives him a utility of zero. An individual will refuse to participate in the mechanism if he does not get an ex-post utility of at least zero.

A queueing problem is said to be $\mathrm{FB}^{*}$ implementable if it is FB implementable by a mechanism which satisfies individual rationality.

PROPOSITION 6.6 $\Omega=\langle\mathbf{N}, \overline{\mathbf{\Theta}}\rangle$ satisfying CP and IP is not FB* implementable.

PROOF: Consider a state $\theta$ satisfying $\mathrm{CP}$ and IP such that $\theta_{j}(1)>v_{j}$ for all $j \in \mathbf{N}$. Clearly,

$$
U_{j}\left(\sigma_{j}^{*}(\theta), t_{j}(\theta), \theta_{j}\right)-t_{j}(\theta)=v_{j}-\theta_{j}\left(\sigma_{j}^{*}(\theta)\right)<0
$$

for all $j \in \mathbf{N}$. Balancedness imply $\sum_{j \in \mathbf{N}} t_{j}(\theta)=0$. If $t_{j}(\theta)<0$ for some $j \in S \subset \mathbf{N}$ then $U_{j}\left(\sigma_{j}^{*}(\theta), t_{j}(\theta), \theta_{j}\right)<0$ for all $j \in S$ and hence individual rationality is not satisfied. So for all $j \in \mathbf{N}, t_{j}(\theta) \geq 0$. Therefore, for balancedness we need $t_{j}(\theta)=0$ for all $j \in \mathbf{N}$. If $t_{j}(\theta)=0$ for all $j \in \mathbf{N}$ then $U_{j}\left(\sigma_{j}^{*}(\theta), t_{j}(\theta), \theta_{j}\right)<0$ for all $j \in \mathbf{N}$. Thus $\Omega=\langle\mathbf{N}, \overline{\boldsymbol{\Theta}}\rangle$ is not $\mathrm{FB}^{*}$ implementable.

The general queueing model is not $\mathrm{FB}^{*}$ implementable simply because the cost of an individual can be so high as to exceed his benefit from the 
service. However, for $\Omega^{G} \in \Omega^{G}$ and separable cost queueing models where an individual's cost parameter $\theta_{j}$ belongs to an interval $\Theta \equiv[\underline{\theta}, \bar{\theta}]$, one can find sufficient condition under which $\mathrm{FB}^{*}$ implementability can be achieved. To establish this result for $\Omega^{G} \in \Omega^{G}$, consider $\bar{\beta}_{j} \geq \beta_{j}(\theta)$ for all $\theta \in \Theta \equiv[\underline{\theta}, \bar{\theta}]$ and for all $j \in \mathbf{N}$. Let $\bar{\beta} \geq \bar{\beta}_{j}$ and let $\underline{v} \leq v_{j}$ for all $j \in \mathbf{N}$.

PROPOSITION 6.7 If $\Omega^{G} \in \Omega^{G}$ satisfies

$$
\underline{v} \geq \sum_{p=1}^{M}\left\{\left[g^{p}(\bar{\theta})-\frac{n-1}{n} g^{p}(\underline{\theta})\right] \sum_{r=1}^{n} f^{p}(r)\right\}+\bar{\beta},
$$

then it is $\mathrm{FB}^{*}$ implementable.

PROOF: Consider $\Omega^{G} \in \Omega^{G}$ and also the Groves mechanism $M$ with the Groves transfer obtained from condition (4.12). Further we take an individual with queue position $k \in\{1, \ldots, n\}$ in state $\theta$. The utility of the individual is given by

$$
\begin{array}{rl} 
& U_{\mu(k)}\left(k, t_{\mu(k)}, \theta_{\mu(k)}\right) \\
= & v_{\mu(k)}-\sum_{p=1}^{M} f^{p}(k) g^{p}\left(\theta_{\mu(k)}\right)-\beta_{\mu(k)}\left(\theta_{\mu(k)}\right)+t_{\mu(k)}(\theta) \\
= & v_{\mu(k)}-\sum_{p=1}^{M} f^{p}(k) g^{p}\left(\theta_{\mu(k)}\right)-\sum_{p=1}^{M} \sum_{r \neq k} f^{p}(r) g^{p}\left(\theta_{\mu(r)}\right)-\beta_{\mu(k)}\left(\theta_{\mu(k)}\right) \\
& \quad+(n-1) \sum_{p=1}^{M}\left\{\sum_{q<k} h^{p}(q) g^{p}\left(\theta_{\mu(q)}\right)+\sum_{q>k} h^{p}(q-1) g^{p}\left(\theta_{\mu(q)}\right)\right\} \\
\geq & v_{\mu(k)}-\sum_{p=1}^{M} g^{p}(\bar{\theta}) \sum_{r=1}^{n} f^{p}(r)+(n-1) \sum_{p=1}^{M} g^{p}(\underline{\theta}) \sum_{r=1}^{n-1} h^{p}(r)-\bar{\beta} \\
= & v_{\mu(k)}-\sum_{p=1}^{M} g^{p}(\bar{\theta}) \sum_{r=1}^{n} f^{p}(r)+\frac{n-1}{n} \sum_{p=1}^{M} g^{p}(\underline{\theta}) \sum_{r=1}^{n} f^{p}(r)-\bar{\beta}(\text { from }(3.8)) \\
= & v_{\mu(k)}-\sum_{p=1}^{M}\left\{g^{p}(\bar{\theta})-\frac{n-1}{n} g^{p}(\underline{\theta})\right\} \sum_{r=1}^{n} f^{p}(r)-\bar{\beta} \\
\geq & \underline{v}-\left[\sum_{p=1}^{M}\left\{g^{p}(\bar{\theta})-\frac{n-1}{n} g^{p}(\underline{\theta})\right\} \sum_{r=1}^{n} f^{p}(r)+\bar{\beta}\right] \\
\geq 0 & 0 .
\end{array}
$$

A similar result follows for the first best implementable separable cost queueing model. 
Corollary 6.1 If a FB implementable $\hat{\Omega}=\langle\mathbf{N}, \Theta,(f, g)\rangle$ satisfies

$$
\underline{v} \geq\left\{\left[g(\bar{\theta})-\frac{n-1}{n} g(\underline{\theta})\right] \sum_{r=1}^{n} f(r)\right\},
$$

then it is $\mathrm{FB}^{*}$ implementable.

The prove of this corollary is immediate from the fact that a first best implementable separable cost queueing model is a special case of $\Omega^{G}$ with $M=1$ and $\beta_{j}(\theta)=0$ for all $\theta_{j} \in \Theta$ and for all $j \in \mathbf{N}$.

From this section one can conclude that with a very general cost structure one cannot $\mathrm{FB}^{*}$ implement the queueing model. However, for the separable cost queueing models and general FB implementable class of queueing models one can find lower bounds on the benefit, sufficient for FB* implementability.

\section{Conclusion}

This paper provides a complete characterization of FB implementability of queueing models. For a queueing model to be first best implementable, it is necessary and sufficient that the type vector of each individual satisfies a certain combinatorial property called $\mathrm{CP}$ and that the externality that can be imposed by an individual on the remaining set of individuals satisfies the independence property. The class of queueing models analysed in this paper are implementable only by Groves mechanism. Therefore, the first best implementability of a queueing problem reduces to the problem of finding appropriate balanced Groves mechanism. The independence property allows for a particular type of separability which matches the separability obtained from the combinatorial property. This paper identifies a fairly large class of first best implementable queueing models. For completeness, the question of individual rationality of the FB implementable queueing models is analysed in section six. The broad conclusion from this analysis is that if the benefit derived by each individual from the service is sufficiently high, then a FB implementable queueing model satisfies individual rationality. 


\section{REFERENCES}

Bapat, R. B., and Raghavan, T. E. S. (1997). Non-negative Matrices and Applications. Cambridge University Press.

Clarke, E. H. (1971). "Multi-part Pricing of Public Goods," Public Choice 11, 17-33.

Green, J., and Laffont, J. J. (1977). "Characterization of Satisfactory Mechanisms for the Revelation of Preferences for Public Goods," Econometrica 45, 427-438.

Green, J., and Laffont, J. J. (1979). Incentives in Public Decision Making. North Holland Publication, Amsterdam.

Groves, T., (1973). "Incentives in Teams," Econometrica 41, 617-631.

Holmström, B. (1977). "On Incentives and Control in Organisation", Ph.D Dissertation, Stanford Graduate School of Business.

Holmström, B. (1979). "Groves' Schemes on Restricted Domains," Econometrica 47, 1137-1144.

Mitra, M., and Sen, A. (1998). "Implementation of first best public decisions in dominant strategies," mimeo: Indian Statistical Institute, New Delhi.

Suijs, J. (1996). "On Incentive Compatibility and Budget Balancedness in Public Decision Making," Economic Design 2, 193-209.

Tomescu, I. (1985). "Problems in Combinatorics and Graph Theory", translated by R.A. Melter, A Wiley-Interscience Publication.

Vickrey, W. (1961). "Counterspeculation, Auctions and Competitive Sealed Tenders," Journal of Finance 16, 8-37.

Walker, M. (1980). "On the Non-Existence of Dominant Strategy Mechanisms for Making Optimal Public Decisions," Econometrica 48, 1521-1540. 